\title{
Transmit Power Allocation for Successive Interference Cancellation in Multicode MIMO Systems
}

\author{
Chang Soon Park, Member, IEEE, and Kwang Bok Lee, Senior Member, IEEE
}

\begin{abstract}
Multiple-input multiple-output (MIMO) system with multicode transmission can provide high speed data services by transmitting independent parallel substreams from multiple antennas and through multicode channelization. In this paper, we first introduce an iterative two-stage successive interference cancellation (SIC) detection scheme for a multicode MIMO system. The proposed technique cancels the interference signals successively in the space domain followed by the code domain. Next, we develop various transmit power allocation schemes over different data substreams for the proposed detection process to improve error rate performance. The joint transmit power allocation is derived to make the post-detection signal-to-interferenceplus-noise ratio (SINR) become the same for all substreams in both the space and code domains. As a computationally efficient scheme, we propose a two-stage power allocation scheme, which allocates the total transmit power to the substreams in the code domain at the first stage, and allocates this code domain power to the substreams in the space domain at the second stage. Furthermore, variable and constant power ratio (PR) schemes are derived to reduce the feedback overhead. In particular, the constant PR scheme utilizes the transmit power ratio determined by the long-term statistical properties of the fading channel amplitudes, and achieves significantly reduced feedback rate. Numerical results show that the proposed transmit power allocation schemes for the two-stage SIC significantly outperform the equal power allocation scheme.
\end{abstract}

Index Terms-Bit error rate (BER), multicode transmission, multiple-input multiple-output (MIMO) systems, successive interference cancellation (SIC), transmit power allocation.

\section{INTRODUCTION}

$\mathbf{T}$ 10 meet increasing demands for wireless data services, higher speed and higher quality transmission is required in next generation wireless communication systems. Multipleinput multiple-output (MIMO) systems, which employ multiple antennas at both the transmitter and receiver, have drawn considerable attention in recent years due to its enormous capacity enhancement [1], [2]. It has been shown that, in rich scattering channel environments, the asymptotic capacity

Paper approved by X. Wang, the Editor for Multiuser Detection and Equalization of the IEEE Communications Society. Manuscript received June 10, 2005; revised November 2, 2006 and July 23, 2007. This paper was presented in part at the IEEE International Conference on Communications, Seoul, Korea, May 2005.

C. S. Park was with the School of Electrical Engineering and Computer Science, Seoul National University, Seoul 151-742, Korea. He is now with Samsung Advanced Institute of Technology, Yongin 446-712, Korea (e-mail: parkcs@mobile.snu.ac.kr)

K. B. Lee is with the School of Electrical Engineering and Computer Science, Seoul National University, Seoul 151-742, Korea (e-mail: klee@snu.ac.kr).

Digital Object Identifier 10.1109/TCOMM.2008.050064 of MIMO Rayleigh fading channels grows linearly with the smaller of the number of transmit and receive antennas. On the other hand, multicode channelization scheme has been proposed for a high rate transmission in a code-division multipleaccess (CDMA) system [3]. The multicode scheme divides a high rate data stream into multiple low rate substreams, and transmits these substreams in parallel using orthogonal spreading codes. In [4], it has been shown that the multicode scheme can achieve a notable performance improvement over the single code scheme in a multipath fading channel. Thus, a MIMO system combined with a multicode transmission, referred to as multicode MIMO system in this paper, may be promising for providing high speed data services [5].

In a frequency-selective fading channel, the orthogonality between two different spreading codes cannot be preserved for the received signals with different path delays. This interference between multicode signals is called multicode interference (MCI) and results in the performance degradation. To mitigate the MCI, various interference suppression techniques may be employed. In [6] and references therein, interference cancellation schemes for multicode CDMA systems have been studied in a single antenna system. In [5], space-time detector based on a group detection technique [7] has been introduced for a cellular MIMO CDMA system with multicode transmission. However, the detection scheme in [5] may cause huge computational complexity when the number of data substreams $D$ is large, since the detection needs a $D \times D$ matrix inversion operation.

In this paper, we first introduce an iterative two-stage successive interference cancellation (SIC) detection scheme for a multicode MIMO system. The first stage successively cancels the interfering signals in the space domain corresponding to a specific spreading code, which essentially follows the conventional vertical Bell Labs layered space-time (VBLAST) detection algorithm [8]. The second stage cancels the interfering signals in the code domain using the previously detected data substreams in the first stage. Compared with the detection scheme in [5], this two-stage approach reduces the matrix dimension for inverse operation, from the number of all the space and code domain substreams (as in [5]), to that of space domain substreams for each code.

Next, we derive various transmit power allocation schemes over different data substreams to improve error rate performance of the proposed two-stage SIC detection process. The power allocation is performed to make the signal-to- 


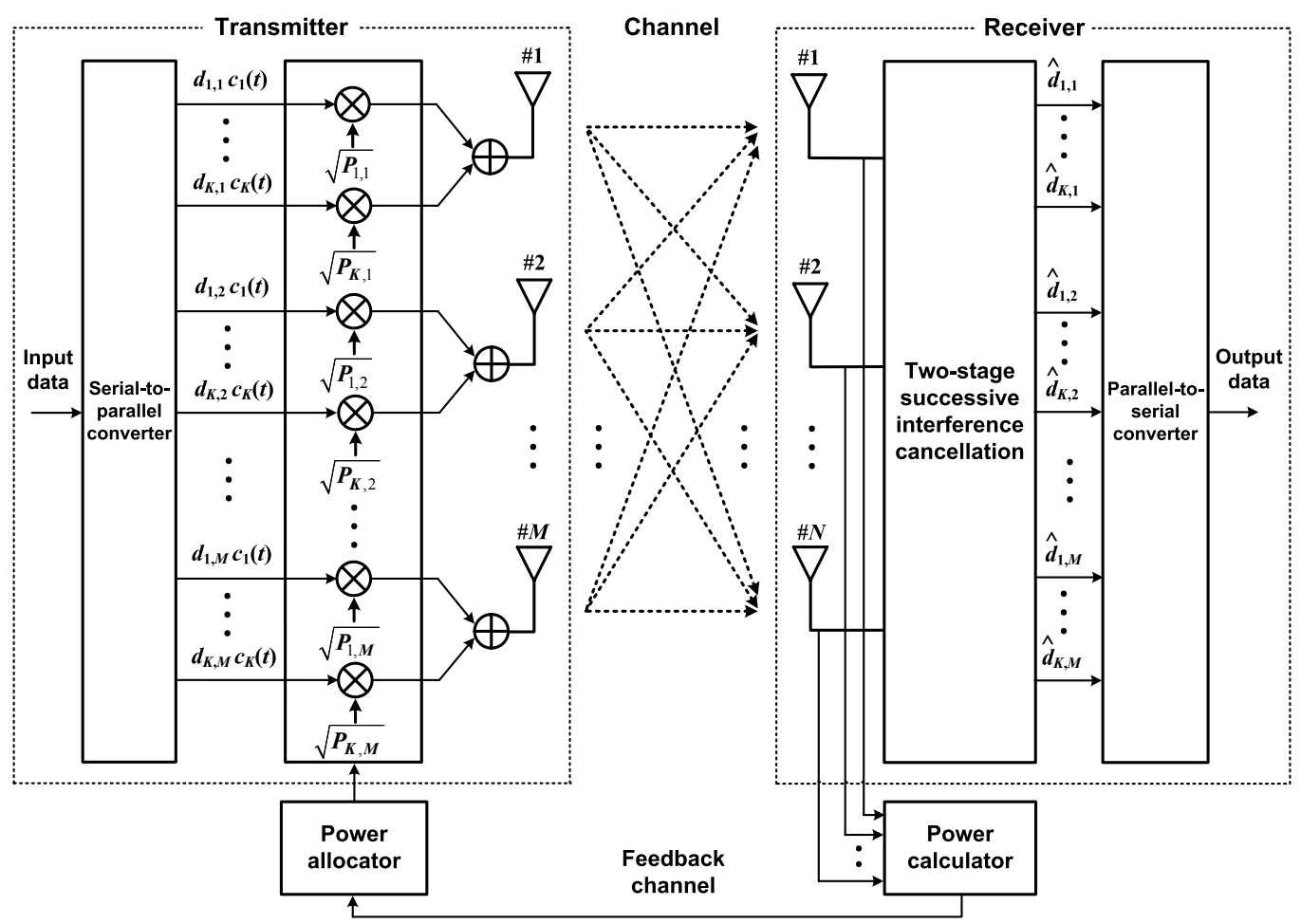

Fig. 1. Multicode MIMO communication system.

interference-plus-noise ratio (SINR) become balanced for all data substreams, based on the notion that the substream with the smallest SINR may dominate the overall error performance of the system. The joint transmit power allocation is derived to make the post-detection SINR become the same for all substreams in both space and code domains. As a computationally efficient scheme, we also propose a two-stage power allocation scheme, which allocates the total transmit power to the substreams in the code domain at the first stage, and this code domain power is allocated to the substreams in the space domain at the second stage. Furthermore, the variable and constant power ratio (PR) schemes are derived to reduce the feedback overhead. In these two schemes, the power allocation requires only "single" power ratio between the two adjacent codes for the feedback information to the transmitter. In particular, the constant PR scheme utilizes the transmit power ratio determined by the long-term statistical properties of the fading channel amplitudes, and achieves significantly reduced feedback rate.

The performance of the proposed detection scheme and power allocation schemes are evaluated, and compared with one another. Numerical results show that the use of the proposed power allocation schemes significantly improves the bit error rate (BER) performance of a multicode MIMO system. The variable and constant PR schemes are found to perform well in the presence of large number of transmit and receive antennas.

The remainder of this paper is organized as follows. Section II describes the system and cannel models. In Section III, the iterative two-stage SIC detection process is derived, and the various transmit power allocation schemes are developed in
Section IV. Numerical results are presented in Section V, and conclusions are drawn in Section VI.

\section{System And Channel Models}

A multicode MIMO communication system considered in this paper is depicted in Fig. 1. We assume that the transmitter and the receiver are equipped with $M$ and $N$ antennas respectively, and $K$ spreading codes are used for multicode channelization under single user environment. An input data stream is divided into $K M$ parallel substreams through a serial-to-parallel converter. The substreams are partitioned into $M$ groups. Each group consists of $K$ data substreams, which are spread using different spreading waveforms $c_{k}(t)$ $(k=1,2, \ldots, K)$ and are transmitted from the same transmit antenna. The spreading waveforms are reused for the substreams at all the other transmit antennas. Hence, the complex baseband transmitted signal of the $m$ th transmit antenna for one data symbol duration may be expressed as

$$
\begin{aligned}
s_{m}(t)=\sum_{k=1}^{K} \sqrt{P_{k, m}} d_{k, m} c_{k}(t), \\
\\
\quad m=1,2, \ldots, M, \quad 0 \leq t \leq T
\end{aligned}
$$

where $P_{k, m}$ is the transmit power for the $k$ th spreading code at the $m$ th transmit antenna, $d_{k, m}$ is the encoded data symbol with unit average power, and $T$ is the symbol duration. It is assumed that a quadrature phase-shift keying (QPSK) is employed for a modulation scheme. Hence, the complex data symbol $d_{k, m}$ is given by $d_{k, m}=d_{k, m}^{(I)}+j d_{k, m}^{(Q)}$, where independent $\left\{d_{k, m}^{(I)}\right\}$ and $\left\{d_{k, m}^{(Q)}\right\}$ take on the values of $+1 / \sqrt{2}$ and $-1 / \sqrt{2}$ with equal probability. $c_{k}(t)$ is the spreading 
waveform for the $k$ th code given as

$$
c_{k}(t)=\sum_{i=0}^{G-1} c_{k, i} \psi\left(t-i T_{c}\right), \quad k=1,2, \ldots, K
$$

where $G=T / T_{c}$ is the spreading gain, $T_{c}$ is the chip duration, $c_{k, i}$ is the $i$ th chip for the $k$ th code, and $\psi(t)$ is the chip pulse shape which is assumed to be rectangular, i.e., one for $0 \leq t \leq$ $T_{c}$ and zero otherwise. The chip sequence $\left\{c_{k, i}\right\}$ is assumed to be a complex spreading sequence, and is given by $c_{k, i}=c_{k, i}^{(I)}+$ $j c_{k, i}^{(Q)}$, where $\left\{c_{k, i}^{(I)}\right\}$ and $\left\{c_{k, i}^{(Q)}\right\}$ take on the random values of $+1 / \sqrt{2}$ and $-1 / \sqrt{2}$ with equal probability. Moreover, $\left\{c_{k, i}\right\}$ are mutually orthogonal for all $k$, i.e., $\sum_{i=0}^{G-1} c_{k_{1}, i} c_{k_{2}, i}^{*}=0$ for $k_{1} \neq k_{2}$, where the superscript * denotes the complex conjugate. The total transmit power constraint is given as

$$
\sum_{k=1}^{K} P_{k}=P_{T}
$$

where $P_{k}=\sum_{m=1}^{M} P_{k, m}$ denotes the sum of power components for the $k$ th spreading code.

The signals between different receive and transmit antennas are assumed to experience frequency-selective and slow fading independently. We model the channel response as the tapped delay line multipath channel model [9]. Thus, the complex channel impulse response from the $m$ th transmit antenna to the $p$ th receive antenna may be expressed as

$$
h_{p, m}(t)=\sum_{l=0}^{L-1} h_{p, m, l} \delta\left(t-l T_{c}\right)
$$

where $L$ is the number of resolvable multipath components. $h_{p, m, l}$ denotes the fading coefficient from the $m$ th transmit antenna to the $p$ th receive antenna for the $l$ th multipath, and they are assumed to be circularly symmetric complex Gaussian random variables with zero mean. It is assumed that all $h_{p, m, l}$ 's are constant over several symbol duration, and are independent for all $p, m$, and $l$. The second moment of channel amplitude $\left|h_{p, m, l}\right|, \Omega_{l}$, is assumed to have the exponential multipath intensity profile (MIP) as

$$
\Omega_{l}=E\left[\left|h_{p, m, l}\right|^{2}\right]=\Omega_{0} e^{-l \delta}, \quad l=0,1, \ldots, L-1
$$

where $E[\cdot]$ denotes the expectation and the parameter $\delta$ represents the rate of the exponential decay of the average path power. The small delay spread relative to the symbol interval is assumed, so that the effect of the intersymbol interference (ISI) on the error performance is negligible compared to that of the intrasymbol interference. Under this assumption, the ISI is ignored in this paper.

Correspondingly, the received complex baseband equivalent signal at the $p$ th receive antenna may be expressed as

$$
\begin{array}{r}
r_{p}(t)=\sum_{m=1}^{M} \sum_{l=0}^{L-1} h_{p, m, l} s_{m}\left(t-l T_{c}\right)+w_{p}(t) \\
=\sum_{k=1}^{K} \sum_{m=1}^{M} \sum_{l=0}^{L-1} \sqrt{P_{k, m}} h_{p, m, l} d_{k, m} c_{k}\left(t-l T_{c}\right)+w_{p}(t), \\
p=1,2, \ldots, N
\end{array}
$$

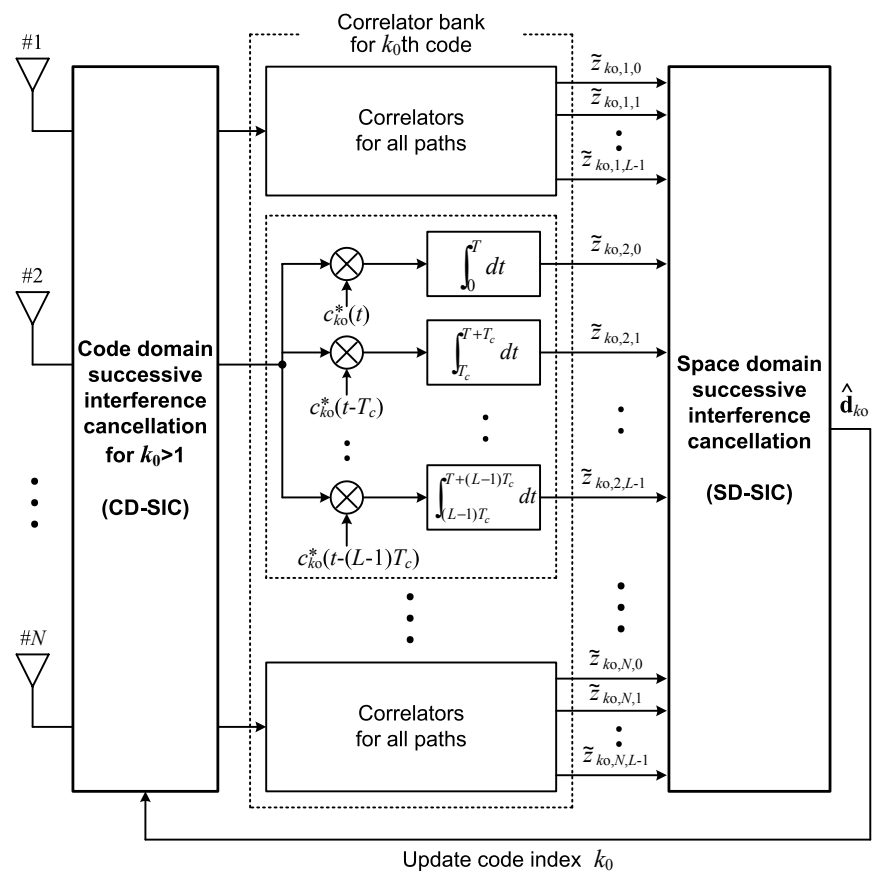

Fig. 2. Two-stage SIC receiver structure.

where $w_{p}(t)$ is the additive white Gaussian noise (AWGN) at the $p$ th receive antenna with one-sided power spectral density $\sigma^{2}$. Throughout this paper, the superscripts $[\cdot]^{T}$ and $[\cdot]^{H}$ denote the transpose and conjugate transpose, respectively. Moreover, $(\mathbf{A})_{i, j}$ denotes the element in the $i$ th row and $j$ th column of a matrix $\mathbf{A}$, and $\|\cdot\|$ represents the Euclidean norm of a vector.

The channel state information (CSI) is estimated at the receiver, and assumed to be perfectly known at the receiver. As depicted in Fig. 1, the transmit power values are calculated at the receiver using the CSI, and fed back to the transmitter through the feedback channel.

\section{Detection PRocess}

The proposed SIC detection process for the multicode MIMO system is described in this section. The overall receiver structure is depicted in Fig. 2. In Section III-A, we describe the output signal of the correlator bank for an arbitrary spreading code, when the SIC processing is not applied yet. In Section III-B, we describe an iterative two-stage SIC detection scheme.

\section{A. Output Signal of a Correlator Bank Without SIC Process- ing}

Prior to the SIC processing, we describe the overall output signal of the correlator bank for the $k_{0}$ th spreading code, which is assumed to be the first code for signal detection. As shown in Fig. 2, the correlator bank for a specific spreading code consists of the $L N$ rake fingers, which despread the received signal for $L$ resolvable multipaths at $N$ receive antennas. The correlator output of the $l$ th rake finger at the $p$ th receive antenna for the $k_{0}$ th spreading code, $z_{k_{0}, p, l}$ $\left(k_{0}=1,2, \ldots, K ; p=1,2, \ldots, N ; l=0,1, \ldots, L-1\right)$, 
may be written as

$$
z_{k_{0}, p, l}=\int_{l T_{c}}^{T+l T_{c}} r_{p}(t) c_{k_{0}}^{*}\left(t-l T_{c}\right) d t .
$$

Then, the NL-dimensional correlator output vector for the $k_{0}$ th spreading code, $\mathbf{z}_{k_{0}}$, may be expressed as

$$
\begin{aligned}
\mathbf{z}_{k_{0}} & \triangleq\left[z_{k_{0}, 1,0}, z_{k_{0}, 1,1}, \ldots, z_{k_{0}, 1, L-1}, z_{k_{0}, 2,0}, \ldots, z_{k_{0}, N, L-1}\right]^{T} \\
& =\mathbf{Y}_{k_{0}, k_{0}} \mathbf{P}_{k_{0}} \mathbf{d}_{k_{0}}+\sum_{k=1, k \neq k_{0}}^{K} \mathbf{Y}_{k_{0}, k} \mathbf{P}_{k} \mathbf{d}_{k}+\mathbf{n}_{k_{0}} .
\end{aligned}
$$

Here, $\mathbf{Y}_{k_{0}, k}=\left[\mathbf{Y}_{k_{0}, k, 1}^{T}, \mathbf{Y}_{k_{0}, k, 2}^{T}, \ldots, \mathbf{Y}_{k_{0}, k, N}^{T}\right]^{T}$ represents the $N L \times M$ space-time code correlation matrix, where $\mathbf{Y}_{k_{0}, k, p}(p=1,2, \ldots, N)$ is the $L \times M$ matrix with elements $\left(\mathbf{Y}_{k_{0}, k, p}\right)_{i, j}=\sum_{l=0}^{L-1} h_{p, j, l} R_{k_{0}, k}(l-(i-1))$, shown in (9) at the bottom of the page, and

$$
R_{k_{0}, k}(l) \triangleq \int_{-\infty}^{\infty} c_{k_{0}}^{*}(t) c_{k}\left(t-l T_{c}\right) d t
$$

denotes the crosscorrelation between the $k_{0}$ th and $k$ th spreading code waveforms when the relative delay is $l$ chips duration. $\mathbf{P}_{k_{0}}=\operatorname{diag}\left(\sqrt{P_{k_{0}, 1}}, \sqrt{P_{k_{0}, 2}}, \ldots, \sqrt{P_{k_{0}, M}}\right)$ is the $M \times M$ power matrix, $\mathbf{d}_{k_{0}}=\left[d_{k_{0}, 1}, d_{k_{0}, 2}, \ldots, d_{k_{0}, M}\right]^{T}$ is the $M \times 1$ data symbol vector, and $\mathbf{n}_{k_{0}}=$ $\left[n_{k_{0}, 1,0}, n_{k_{0}, 1,1}, \ldots, n_{k_{0}, 1, L-1}, n_{k_{0}, 2,0}, \ldots, n_{k_{0}, N, L-1}\right]^{T}$ is the $N L \times 1$ noise vector for the $k_{0}$ th code where $\quad n_{k_{0}, p, l}=\int_{l T_{c}}^{T+l T_{c}} w_{p}(t) c_{k_{0}}^{*}\left(t-l T_{c}\right) d t$. $\sum_{k=1, k \neq k_{0}}^{K} \mathbf{Y}_{k_{0}, k} \mathbf{P}_{k} \mathbf{d}_{k}+\mathbf{n}_{k_{0}}$ in (8) is the MCI-plusnoise vector for the $k_{0}$ th code whose covariance matrix may be expressed as

$$
\begin{gathered}
E\left[\left(\sum_{k=1, k \neq k_{0}}^{K} \mathbf{Y}_{k_{0}, k} \mathbf{P}_{k} \mathbf{d}_{k}+\mathbf{n}_{k_{0}}\right) \times\right. \\
\left.\left(\sum_{k=1, k \neq k_{0}}^{K} \mathbf{Y}_{k_{0}, k} \mathbf{P}_{k} \mathbf{d}_{k}+\mathbf{n}_{k_{0}}\right)^{H}\right] \\
=\sum_{k=1, k \neq k_{0}}^{K} \mathbf{R}_{k_{0}, k}^{\mathrm{MCI}}+\mathbf{R}_{k_{0}}^{\text {noise }}
\end{gathered}
$$

where $\mathbf{R}_{k_{0}, k}^{\mathrm{MCI}}=E\left[\mathbf{Y}_{k_{0}, k} \mathbf{P}_{k}\left(\mathbf{Y}_{k_{0}, k} \mathbf{P}_{k}\right)^{H}\right]$ is the covariance matrix of the MCI vector due to the interference between the data substreams of the $k_{0}$ th and $k$ th codes, and $\mathbf{R}_{k_{0}}^{\text {noise }}=$ $E\left[\mathbf{n}_{k_{0}} \mathbf{n}_{k_{0}}^{H}\right]$ is the covariance matrix of the noise vector for the $k_{0}$ th code. Although the crosscorrelations between the different spreading waveforms in (10) can be computed at the receiver, we average these values over random orthogonal code sequences to find $\mathbf{R}_{k_{0}, k}^{\mathrm{MCI}}$ in (11) for computational efficiency. The expected values of the product of two code correlation functions in (10) can be found as [10]

$$
\begin{aligned}
& E\left[R_{k_{0}, k}(i) R_{k_{0}, k}^{*}(j)\right] \\
& = \begin{cases}G^{2} T_{c}^{2}, & k_{0}=k, i=j=0 \\
(G-|i|) T_{c}^{2}, & i=j \neq 0,|i| \leq G-1 \\
0, & \text { else. }\end{cases}
\end{aligned}
$$

Using (12), we can find the element in the $i^{\prime}(=L(p-1)+i)$ th row and $j^{\prime}(=L(q-1)+j)$ th column of $\mathbf{R}_{k_{0}, k}^{\mathrm{MCI}}$ as shown in (13) at the bottom of the page. Covariance matrix of noise vector, $\mathbf{R}_{k_{0}}^{\text {noise }}$, can be found as

$$
\mathbf{R}_{k_{0}}^{\text {noise }}=\sigma^{2} \operatorname{diag}\left(\tilde{\mathbf{R}}_{k_{0}}, \tilde{\mathbf{R}}_{k_{0}}, \ldots, \tilde{\mathbf{R}}_{k_{0}}\right)
$$

where $\tilde{\mathbf{R}}_{k_{0}}$ is the $L \times L$ correlation matrix for the spreading waveforms of the $k_{0}$ th code, with elements $\left(\tilde{\mathbf{R}}_{k_{0}}\right)_{i, j}=$ $\int_{-\infty}^{\infty} c_{k_{0}}^{*}\left(t-(i-1) T_{c}\right) c_{k_{0}}\left(t-(j-1) T_{c}\right) d t=R_{k_{0}, k_{0}}(j-i)$.

\section{B. Two-Stage SIC Scheme}

Based on the results in the previous subsection, an iterative two-stage SIC scheme is described in this subsection. In the first stage, space domain SIC (SD-SIC) successively cancels the intracode interference caused by the data substreams from the different transmit antennas for a specific spreading code. Essentially, this processing follows the conventional VBLAST detection algorithm [8]. However, unlike the case of a conventional MIMO system, the signal processing in this paper provides the additional outputs obtained by the rake reception. This multipath diversity reception by rake receiver may be

$$
\mathbf{Y}_{k_{0}, k, p}=\sum_{l=0}^{L-1}\left[\begin{array}{cccc}
h_{p, 1, l} R_{k_{0}, k}(l) & h_{p, 2, l} R_{k_{0}, k}(l) & \cdots & h_{p, M, l} R_{k_{0}, k}(l) \\
h_{p, 1, l} R_{k_{0}, k}(l-1) & h_{p, 2, l} R_{k_{0}, k}(l-1) & \cdots & h_{p, M, l} R_{k_{0}, k}(l-1) \\
\vdots & \vdots & \ddots & \vdots \\
h_{p, 1, l} R_{k_{0}, k}(l-(L-1)) & h_{p, 2, l} R_{k_{0}, k}(l-(L-1)) & \cdots & h_{p, M, l} R_{k_{0}, k}(l-(L-1))
\end{array}\right]
$$

$$
\begin{aligned}
\left(\mathbf{R}_{k_{0}, k}^{\mathrm{MCI}}\right)_{i^{\prime}, j^{\prime}} & =E\left[\sum_{m=1}^{M} P_{k, m}\left(\sum_{l=0}^{L-1} h_{p, m, l} R_{k_{0}, k}(l-(i-1)) \sum_{l^{\prime}=0}^{L-1} h_{q, m, l^{\prime}}^{*} R_{k_{0}, k}^{*}\left(l^{\prime}-(j-1)\right)\right)\right] \\
& =\left\{\begin{array}{r}
\sum_{m=1}^{M} P_{k, m} \sum_{\substack{l=i-j \\
l \neq i-1}}^{L-1} h_{p, m, l} h_{q, m, l-(i-j)}^{*}(G-|l-(i-1)|) T_{c}^{2}, \quad i \geq j \\
\sum_{m=1}^{M} P_{k, m} \sum_{\substack{l=j-i \\
l \neq j-1}}^{L-1} h_{p, m, l-(j-i)} h_{q, m, l}^{*}(G-|l-(j-1)|) T_{c}^{2}, \quad j>i \\
k_{0} \neq k ; p, q=1,2, \ldots, N ; i, j=1,2, \ldots, L
\end{array}\right.
\end{aligned}
$$


regarded as the virtual receive antenna diversity reception. As described in the previous subsection, there are $L$ correlator output signals for rake reception at each receive antenna. In order to utilize these rake output signals for SD-SIC, it is assumed that, at each receive antenna, there are $L-1$ virtual antennas as well as the actual one. Thus, the dimension of a channel matrix required to detect all data substreams for an arbitrary spreading code is $N L \times M$, not $N \times M$. It is assumed that $N L \geq M$ for signal detection to be possible.

In the second stage for SIC, code domain SIC (CD-SIC) cancels the MCI caused by the nonorthogonality between different spreading codes. In this processing, the contributions of pre-detected code domain signals are subtracted from the received signal after the space domain signals for each code channel are all detected through SD-SIC in the previous stage. If we assume that the detection order of code domain signals is from 1 to $K$, i.e., any particular ordering criterion is not used, the MCI cancelled correlator output of the $l$ th rake finger at the $p$ th receive antenna for the $k_{0}$ th spreading code, $\tilde{z}_{k_{0}, p, l}$ $\left(k_{0}=1,2, \ldots, K ; p=1,2, \ldots, N ; l=0,1, \ldots, L-1\right)$, may be written as

$$
\begin{aligned}
\tilde{z}_{k_{0}, p, l}= & \int_{l T_{c}}^{T+l T_{c}}\left(r_{p}(t)-\sum_{k=1}^{k_{0}-1} \sum_{m=1}^{M} \sum_{i=0, i \neq l}^{L-1} \sqrt{P_{k, m}}\right. \\
& \left.\times h_{p, m, i} \hat{d}_{k, m} c_{k}\left(t-i T_{c}\right)\right) c_{k_{0}}^{*}\left(t-l T_{c}\right) d t
\end{aligned}
$$

where $\hat{d}_{k, m}$ is the hard estimate for the data symbol $d_{k, m}$ in the previous SD-SIC detection stage. Then, the MCI cancelled $N L$-dimensional correlator output vector for the $k_{0}$ th spreading code, $\tilde{\mathbf{z}}_{k_{0}}$, may be expressed as

$$
\begin{aligned}
\tilde{\mathbf{z}}_{k_{0}} \triangleq & {\left[\tilde{z}_{k_{0}, 1,0}, \tilde{z}_{k_{0}, 1,1}, \ldots, \tilde{z}_{k_{0}, 1, L-1}, \tilde{z}_{k_{0}, 2,0}, \ldots, \tilde{z}_{k_{0}, N, L-1}\right]^{T} } \\
= & \mathbf{Y}_{k_{0}, k_{0}} \mathbf{P}_{k_{0}} \mathbf{d}_{k_{0}}+\sum_{k=1, k \neq k_{0}}^{K} \mathbf{Y}_{k_{0}, k} \mathbf{P}_{k} \mathbf{d}_{k} \\
& -\sum_{k=1}^{k_{0}-1} \mathbf{Y}_{k_{0}, k} \mathbf{P}_{k} \hat{\mathbf{d}}_{k}+\mathbf{n}_{k_{0}}
\end{aligned}
$$

where $\hat{\mathbf{d}}_{k}=\left[\hat{d}_{k, 1}, \hat{d}_{k, 2}, \ldots, \hat{d}_{k, M}\right]^{T}$ is the estimated data symbol vector for the $k$ th code. Note that the receiver can obtain all elements of $\tilde{\mathbf{z}}_{k_{0}}$ without explicit computation of $R_{k_{0}, k}(l)$ 's in (10) for $k_{0} \neq k$, as indicated in (15).

This two-stage SIC process described above is recursively performed until all data symbols are detected. The overall detection procedure can be presented as follows. For simplicity, zero-forcing (ZF) criterion is used to calculate the linear weighting vector of SD-SIC.

Step 1) Initialization for CD-SIC:

$$
\begin{aligned}
& k_{0}=1 \\
& \tilde{\mathbf{z}}_{1}=\mathbf{z}_{1}=\mathbf{Y}_{1,1} \mathbf{P}_{1} \mathbf{d}_{1}+\sum_{k=2}^{K} \mathbf{Y}_{1, k} \mathbf{P}_{k} \mathbf{d}_{k}+\mathbf{n}_{1}
\end{aligned}
$$

Step 2) Initialization for SD-SIC:

$$
\begin{aligned}
& i=1 \\
& \tilde{\mathbf{z}}_{k_{0}}(1)=\tilde{\mathbf{z}}_{k_{0}} \\
& \mathbf{G}_{k_{0}}(1)=\left(\mathbf{Y}_{k_{0}, k_{0}}\right)^{\dagger} \\
& g(1)=\underset{j}{\arg \min }\left(\mathbf{G}_{k_{0}}(1) \mathbf{R}_{k_{0}}^{\text {order }} \mathbf{G}_{k_{0}}(1)^{H}\right)_{j, j}
\end{aligned}
$$

Step 3) SD-SIC for the $g(i)$ th substream of the $k_{0}$ th code:

$$
\begin{aligned}
& \mathbf{w}_{k_{0}, g(i)}=\left[\mathbf{G}_{k_{0}}(i)\right]_{g(i)} \\
& \hat{d}_{k_{0}, g(i)}=Q\left(\mathbf{w}_{k_{0}, g(i)} \tilde{\mathbf{z}}_{k_{0}}(i)\right) \\
& \tilde{\mathbf{z}}_{k_{0}}(i+1)=\tilde{\mathbf{z}}_{k_{0}}(i)- \\
& \sqrt{P_{k_{0}, g(i)}} \hat{d}_{k_{0}, g(i)}\left\langle\mathbf{Y}_{k_{0}, k_{0}}\right\rangle_{g(i)}, \quad i<M \\
& \mathbf{G}_{k_{0}}(i+1)=\left(\left\langle\mathbf{Y}_{k_{0}, k_{0}}\right\rangle_{g(i)}\right)^{\dagger}, \quad i<M \\
& g(i+1)=\underset{j \notin\{g(1), \ldots, g(i)\}}{\arg \min }\left(\mathbf{G}_{k_{0}}(i+1) \mathbf{R}_{k_{0}}^{\text {order }}\right. \\
& \left.\quad \times \mathbf{G}_{k_{0}}(i+1)^{H}\right)_{j, j}, \quad i<M
\end{aligned}
$$

Step 4) Repetition or termination for SD-SIC: If $i<M$, increase $i$ by one and go to Step 3. Otherwise, go to Step 5.

Step 5) CD-SIC for the substreams of the $\left(k_{0}+1\right)$ th code $\left(k_{0}<K\right)$ :

$$
\begin{aligned}
\tilde{\mathbf{z}}_{k_{0}+1}= & \mathbf{Y}_{k_{0}+1, k_{0}+1} \mathbf{P}_{k_{0}+1} \mathbf{d}_{k_{0}+1} \\
& +\sum_{k=1, k \neq k_{0}+1}^{K} \mathbf{Y}_{k_{0}+1, k} \mathbf{P}_{k} \mathbf{d}_{k} \\
& -\sum_{k=1}^{k_{0}} \mathbf{Y}_{k_{0}+1, k} \mathbf{P}_{k} \hat{\mathbf{d}}_{k}+\mathbf{n}_{k_{0}+1}
\end{aligned}
$$

Step 6) Repetition or termination for CD-SIC: If $k_{0}<K$, increase $k_{0}$ by one and go to Step 2. Otherwise, terminate the whole detection procedure.

where $(\cdot)^{\dagger}$ denotes the Moore-Penrose pseudo-inverse, $[\cdot]_{g(i)}$ is the $g(i)$ th row of a matrix, and $Q(\cdot)$ is the quantization operation appropriate to the modulation scheme. $\langle\cdot\rangle_{g(i)}$ denotes the $g(i)$ th column of a matrix, and $\langle\cdot\rangle_{g(i)}$ is the deflated version of a matrix, in which columns $g(1), g(2), \ldots, g(i)$ have been zeroed. $\mathbf{R}_{k_{0}}^{\text {order }}$ is a matrix used for SD-SIC detection ordering, and is set to $\sum_{k=k_{0}+1}^{K} \mathbf{R}_{k_{0}, k}^{\mathrm{MCI}}+\mathbf{R}_{k_{0}}^{\text {noise }}$. Note that the detection ordering processes in (17f) and (17k) are performed to select the substream with the largest SINR at each iteration, when interfering signal powers $P_{k, m}$ 's for $k \geq k_{0}+1$ are given and $P_{k_{0}, m}$ 's are assumed to be equal for all $m$. ${ }^{1}$

Let us express the conditional BER for the proposed detection scheme, which will be used for the numerical results in Section V. The decision statistic $\mathbf{w}_{k_{0}, g(i)} \tilde{\mathbf{z}}_{k_{0}}(i)$ in (17h) may be considered as a complex Gaussian random variable with mean $\mathbf{w}_{k_{0}, g(i)}\left(\tilde{\mathbf{z}}_{k_{0}}(i)-\mathbf{n}_{k_{0}}\right)$ and variance

\footnotetext{
${ }^{1}$ The performance degradation can be shown to be negligible, even when the matrix $\mathbf{R}_{k_{0}}^{\text {order }}$ is set to identity matrix for computational simplicity, although the related simulation results are not included in this paper.
} 
$\mathbf{w}_{k_{0}, g(i)} \mathbf{R}_{k_{0}}^{\text {noise }} \mathbf{w}_{k_{0}, g(i)}^{H}$, when all the components of $\mathbf{w}_{k_{0}, g(i)}$ and $\tilde{\mathbf{z}}_{k_{0}}(i)$ except for $\mathbf{n}_{k_{0}}$ are given. When the real part of the transmitted data symbol is assumed to be positive, the conditional BER for the real part of the $g(i)$ th substream of the $k_{0}$ th code may be expressed as

$$
\operatorname{BER}_{k_{0}, g(i)}^{\mathrm{re}}=\frac{1}{2} \operatorname{erfc}\left(\frac{\operatorname{Re}(S)}{\sqrt{\mathbf{w}_{k_{0}, g(i)} \mathbf{R}_{k_{0}}^{\text {noise }} \mathbf{w}_{k_{0}, g(i)}^{H}}}\right)
$$

where $\operatorname{erfc}(x) \triangleq(2 / \sqrt{\pi}) \int_{x}^{\infty} \exp \left(-t^{2}\right) d t$ denotes the complementary error function, $\operatorname{Re}(\cdot)$ denotes the real part, and

$$
\begin{aligned}
& S=\mathbf{w}_{k_{0}, g(i)}\left(\mathbf{Y}_{k_{0}, k_{0}} \mathbf{P}_{k_{0}} \mathbf{d}_{k_{0}}+\sum_{k=1, k \neq k_{0}}^{K} \mathbf{Y}_{k_{0}, k} \mathbf{P}_{k} \mathbf{d}_{k}-\right. \\
& \left.\sum_{k=1}^{k_{0}-1} \mathbf{Y}_{k_{0}, k} \mathbf{P}_{k} \hat{\mathbf{d}}_{k}-\sum_{m=1}^{i-1} \sqrt{P_{k_{0}, g(m)}} \hat{d}_{k_{0}, g(m)}\left\langle\mathbf{Y}_{k_{0}, k_{0}}\right\rangle_{g(m)}\right) .
\end{aligned}
$$

In the detection process described above, any particular detection ordering for CD-SIC is not applied. The reason for this is that all data substreams for all spreading codes undergo the same channel responses between arbitrary transmit and receive antennas, and hence the detection ordering in the code domain is expected to have negligible effect on the BER performance. $^{2}$

\section{Transmit Power Allocation}

When the two-stage SIC process described above is performed with an equal power distribution, the SINRs for all data substreams, and thus the BER, may be widely different. In particular, the data substreams for the earlier detected code index may provide the smallest SINRs and limit the overall error rate performance. In order to overcome this performance limitation, the total transmit power may be allocated in a more effective way to achieve better BER performance. However, the optimal power allocation scheme, which minimizes the overall BER, is quite difficult to find, since the SINR expressed as a complicated matrix form in Section IV-A is not tractable. Thus, in this section, we develop several effective transmit power allocation schemes based on the equal SINR design, which is a feasible approach to improve error rate performance. The equal SINR design balances the SINR for all data substreams, and thus improves the smallest SINR in the equal power distribution case, which may dominate the overall BER performance. The works in [11]-[13] use this design for SIC receiver in CDMA, multicarrier CDMA, and MIMO systems, respectively. However, the detailed problem formulations of those works seem to be considerably different from that in a multicode MIMO system considered in this paper.

The joint power allocation scheme, which makes the postdetection SINR become the same for all substreams in both space and code domains, is developed in Section IV-A. The

\footnotetext{
${ }^{2}$ This negligible performance degradation can be shown by applying the max-min code ordering at each CD-SIC stage. It selects the code index with the maximum among the SINR values, each one of which corresponds to the minimum SINR in its code domain.
}

two-stage power allocation scheme is derived as a simplified version of the joint power allocation scheme in Section IV-B. The variable and constant PR schemes are developed to reduce the feedback overhead in Section IV-C. Some analytical results of code power ratio derived in Section IV-C are presented in Section IV-D.

\section{A. Joint Power Allocation}

To derive the joint power allocation scheme, we first define the post-detection SINR of the $g(i)$ th substream for the $k_{0}$ th code, $\Gamma_{k_{0}, g(i)}$, as the SINR of the decision statistic $\mathbf{w}_{k_{0}, g(i)} \tilde{\mathbf{z}}_{k_{0}}(i)$ in $(17 \mathrm{~h})$ when the error propagation is ignored, i.e.,

$$
\begin{aligned}
\Gamma_{k_{0}, g(i)} \triangleq & P_{k_{0}, g(i)} / \Lambda_{k_{0}, g(i)}, \\
& k_{0}=1,2, \ldots, K ; \quad g(i)=1,2, \ldots, M
\end{aligned}
$$

where $\Lambda_{k_{0}, g(i)} \triangleq \mathbf{w}_{k_{0}, g(i)}\left(\sum_{k=k_{0}+1}^{K} \mathbf{R}_{k_{0}, k}^{\mathrm{MCI}}+\mathbf{R}_{k_{0}}^{\text {noise }}\right) \mathbf{w}_{k_{0}, g(i)}^{H}$ denotes the variance of MCI-plus-noise component. As shown in (13), the post-detection SINRs for the $k_{0}$ th code are functions of the transmit antenna powers for $K-k_{0}$ residual interfering codes as well as the desired code. For large $K$, it may be difficult to find a closed-form solution for the post-detection SINRs to be equal for all substreams in both space and code domains. In this case, an adaptive method may be employed to find a solution in an iterative manner as follows.

Step 1) Initialization for Loop 1: Set an iteration number $a=1$ and an arbitrary initial $K$ th code power $P_{K}(1)\left(0<P_{K}(1)<P_{T}\right)$.

Step 2) Initialization for Loop 2 :

$$
k_{0}=K
$$

Step 3) Initialization for Loop 3:

$$
\begin{aligned}
& i=1 \\
& \mathbf{G}_{k_{0}}(1)=\left(\mathbf{Y}_{k_{0}, k_{0}}\right)^{\dagger} \\
& g(1)=\underset{j}{\arg \min }\left(\mathbf{G}_{k_{0}}(1) \mathbf{R}_{k_{0}}^{\text {order }}(a) \mathbf{G}_{k_{0}}(1)^{H}\right)_{j, j}
\end{aligned}
$$

Step 4) Calculation of MCI-plus-noise variance $\Lambda_{k_{0}, g(i)}(a)$ for the $g(i)$ th substream of the $k_{0}$ th code:

$$
\begin{aligned}
& \mathbf{w}_{k_{0}, g(i)}=\left[\mathbf{G}_{k_{0}}(i)\right]_{g(i)} \\
& \Lambda_{k_{0}, g(i)}(a)=\mathbf{w}_{k_{0}, g(i)} \times \\
& \left(\sum_{k=k_{0}+1}^{K} \mathbf{R}_{k_{0}, k}^{\mathrm{MCI}}(a)+\mathbf{R}_{k_{0}}^{\text {noise }}\right) \mathbf{w}_{k_{0}, g(i)}^{H} \quad(21 \mathrm{f}) \\
& \mathbf{G}_{k_{0}}(i+1)=\left(\left\langle\mathbf{Y}_{k_{0}, k_{0}}\right\rangle \overline{g(i)}\right)^{\dagger}, \quad i<M \quad(21 \mathrm{~g}) \\
& g(i+1)=\underset{j \notin\{g(1), \ldots, g(i)\}}{\arg \min }\left(\mathbf{G}_{k_{0}}(i+1) \mathbf{R}_{k_{0}}^{\text {order }}(a)\right. \\
& \left.\quad \times \mathbf{G}_{k_{0}}(i+1)^{H}\right)_{j, j}, \quad i<M \quad(21 \mathrm{~h})
\end{aligned}
$$

Step 5) Repetition or termination for Loop 3: If $i<M$, increase $i$ by one and go to Step 4. If $i=M$ and $k_{0}=K$, go to Step 6. Otherwise, go to Step 7 . 
Step 6) Adjustment of the reference SINR $\Gamma^{\mathrm{ref}}(a)$ for all data substreams:

$$
\begin{aligned}
\Gamma^{\mathrm{ref}}(a) & =\frac{P_{K}(a)}{\sum_{m=1}^{M} \Lambda_{K, m}(a)} \\
& =\frac{P_{K}(a)}{\sum_{m=1}^{M} \mathbf{w}_{K, m} \mathbf{R}_{K}^{\text {noise }} \mathbf{w}_{K, m}^{H}}
\end{aligned}
$$

Step 7) Calculation of the transmit power set for the $k_{0}$ th code, $\left\{P_{k_{0}, m}(a) \mid m=1,2, \ldots, M\right\}$ :

$$
\begin{aligned}
P_{k_{0}}(a)= & \Gamma^{\mathrm{ref}}(a) \sum_{m=1}^{M} \Lambda_{k_{0}, m}(a) \\
P_{k_{0}, m}(a) & =\Lambda_{k_{0}, m}(a) \frac{P_{k_{0}}(a)}{\sum_{i=1}^{M} \Lambda_{k_{0}, i}(a)} \\
& =\Lambda_{k_{0}, m}(a) \Gamma^{\mathrm{ref}}(a)
\end{aligned}
$$

Step 8) Repetition or termination for Loop 2: If $k_{0}>1$, decrease $k_{0}$ by one and go to Step 3. Otherwise, go to Step 9.

Step 9) Repetition or termination for Loop 1: If $\left|\sum_{k=1}^{K} P_{k}(a)-P_{T}\right|>\varepsilon$, update the $K$ th code power $P_{K}(a)$ as

$P_{K}(a+1)= \begin{cases}P_{K}(a)-\Delta, & \text { if } \sum_{k=1}^{K} P_{k}(a)>P_{T} \\ P_{K}(a)+\Delta, & \text { otherwise }\end{cases}$

where $\varepsilon(\varepsilon>0)$ is a maximum allowable error of the sum of all updated power components, and $\Delta$ $(\Delta>0)$ is a step size for $P_{K}(a)$. Then, increase $a$ by one, and go to Step 2. Otherwise, terminate the whole joint power allocation algorithm.

Note that the iterative procedure described above obtains the power components for each code in the reverse order of code index, since the values of $\Lambda_{k_{0}, g(i)}(a)$ for the $k_{0}$ th code require the knowledge of interfering signal powers $P_{k, m}(a)$ 's for $k \geq k_{0}+1$. Using noise variances $\left\{\mathbf{w}_{K, m} \mathbf{R}_{K}^{\text {noise }} \mathbf{w}_{K, m}^{H} \mid m=1,2, \ldots, M\right\}$ and the given code power $P_{K}(a)$ for the last code $K$, we can calculate the reference post-detection SINR $\Gamma^{\mathrm{ref}}(a)$ and all power components for the code $K$, as shown in (21i) and (21k). Using these computed values, all the other power components can be calculated in the reverse order of code index. When the difference between the sum of all calculated power values and the given total power $P_{T}$ is larger than some threshold, the power allocation procedure is repeated with adjusted value of $P_{K}(a)$. The reason that $P_{K}(a)$ should be decreased if $\sum_{k=1}^{K} P_{k}(a)>P_{T}$ as in $(21 l)$ is as follows. When $P_{K}(a)$ decreases, $\Gamma^{\mathrm{ref}}(a)$ in (21i) decreases and all power values for the $K$ th code $\left\{P_{K, m}(a) \mid m=1,2, \ldots, M\right\}$ decrease with the same scaling factor as shown in $(21 \mathrm{k})$. Therefore, $\Lambda_{K-1, m}(a)=\mathbf{w}_{K-1, m}\left(\mathbf{R}_{K-1, K}^{\mathrm{MCI}}(a)+\mathbf{R}_{K}^{\text {noise }}\right) \mathbf{w}_{K-1, m}^{H}$ decreases for all $m$, and $P_{K-1}(a)$ in $(21 \mathrm{j})$ decreases. In similar manners, all the other code powers decrease. Hence, $\sum_{k=1}^{K} P_{k}(a)$ decreases. From this result, it can be seen that $\sum_{k=1}^{K} P_{k}(a)$ converges to $P_{T}$ by the procedure in $(21 l)$.

It should be noted that all final values for the nulling vector and detection ordering, calculated in the power allocation procedure, can be used in the detection procedure described in Section III without repeating calculations.

\section{B. Two-Stage Power Allocation}

The joint power allocation scheme described in Section IVA is used to provide the equal post-detection SINR for all data substreams in both space and code domains. However, it employs the adaptive method and it generally takes a lot of iterations for an adaptive solution to be close to the optimal one. Thus, its computational complexity may be high. In this subsection, we derive two-stage power allocation scheme as a simplified version of the joint power allocation scheme. The proposed two-stage scheme performs the code domain power allocation, followed by the space domain power allocation. In the first stage, an approximate closed-form solution for the code power set $\left\{P_{k} \mid k=1,2, \ldots, K\right\}$ is derived. In the second stage, based on the values calculated in the first stage, the antenna power components for each code, $\left\{P_{k, m} \mid k=1,2, \ldots, K ; m=1,2, \ldots, M\right\}$, are found in a recursive manner.

1) Code Domain Power Allocation (First Stage): To find the code domain power allocation, we first define the code domain SINR for the $k_{0}$ th code, $\Gamma_{k_{0}}^{\mathrm{CD}}$, as

$$
\begin{array}{r}
\Gamma_{k_{0}}^{\mathrm{CD}} \triangleq \frac{E\left[\left\|\mathbf{Y}_{k_{0}, k_{0}} \mathbf{P}_{k_{0}} \mathbf{d}_{k_{0}}\right\|^{2}\right]}{E\left[\left\|\sum_{k=k_{0}+1}^{K} \mathbf{Y}_{k_{0}, k} \mathbf{P}_{k} \mathbf{d}_{k}+\mathbf{n}_{k_{0}}\right\|^{2}\right]}, \\
k_{0}=1,2, \ldots, K
\end{array}
$$

where the expectations are taken with respect to the spreading sequences as well as the data and noise vectors. As shown in (16), $\mathbf{Y}_{k_{0}, k_{0}} \mathbf{P}_{k_{0}} \mathbf{d}_{k_{0}}$ in (22) is the desired signal vector and $\sum_{k=k_{0}+1}^{K} \mathbf{Y}_{k_{0}, k} \mathbf{P}_{k} \mathbf{d}_{k}+\mathbf{n}_{k_{0}}$ in (22) is the MCI-plusnoise vector with the error propagation ignored. Note that the elements of these vectors are the signals posterior to the CD-SIC detection process and prior to the SD-SIC detection process. Based on the equal code domain SINR design, we allocate the code power so that code domain SINRs in (22) become equal for all codes as

$$
\Gamma_{k_{0}}^{\mathrm{CD}}=\Gamma^{\mathrm{CD}}, \quad k_{0}=1,2, \ldots, K .
$$

Using (12), it can be shown that the equation (23) is calculated as

$$
\begin{aligned}
& \frac{\sum_{m=1}^{M} A(m) P_{k_{0}, m}}{\sum_{k=k_{0}+1}^{K} \sum_{m=1}^{M} B(m) P_{k, m}+\eta}=\Gamma^{\mathrm{CD}}, \\
& k_{0}=1,2, \ldots, K
\end{aligned}
$$

where

$$
\begin{aligned}
A(m) & =\frac{1}{N L} \sum_{p=1}^{N} \sum_{i=1}^{L}\left(\left|h_{p, m,(i-1)}\right|^{2} G^{2} T_{c}^{2}+\right. \\
& \left.\sum_{l=0, l \neq i-1}^{L-1}\left|h_{p, m, l}\right|^{2}(G-|l-(i-1)|) T_{c}^{2}\right) \\
B(m)= & \frac{1}{N L} \sum_{p=1}^{N} \sum_{i=1}^{L} \sum_{\substack{l=0 \\
l \neq i-1}}^{L-1}\left|h_{p, m, l}\right|^{2}(G-|l-(i-1)|) T_{c}^{2}
\end{aligned}
$$




$$
\eta=\sigma^{2} T
$$

If we assume that $N L$ is sufficiently large for a finite value of $L$, we can approximate (25) and (26) to the constant values regardless of $m$, using the law of large numbers [14]. Using this approximation, $A(m)$ and $B(m)$ may be approximated as

$$
\begin{gathered}
A(m) \cong \frac{1}{M N L} \sum_{m=1}^{M} \sum_{p=1}^{N} \sum_{i=1}^{L}\left(\left|h_{p, m,(i-1)}\right|^{2} G^{2} T_{c}^{2}+\right. \\
\left.\sum_{l=0, l \neq i-1}^{L-1}\left|h_{p, m, l}\right|^{2}(G-|l-(i-1)|) T_{c}^{2}\right) \triangleq \alpha \\
B(m) \cong \frac{1}{M N L} \sum_{m=1}^{M} \sum_{p=1}^{N} \sum_{i=1}^{L} \sum_{\substack{l=0 \\
l \neq i-1}}^{L-1}\left|h_{p, m, l}\right|^{2} \\
\times(G-|l-(i-1)|) T_{c}^{2} \triangleq \beta .
\end{gathered}
$$

Then, the equation (24) may be simplified to

$$
\frac{\alpha P_{k_{0}}}{\beta \sum_{k=k_{0}+1}^{K} P_{k}+\eta}=\Gamma^{\mathrm{CD}}, \quad k_{0}=1,2, \ldots, K .
$$

Solving $K+1$ simultaneous equations in (3) and (30) as derived in Appendix A, we can find the code power values as

$$
P_{k}=\left(\frac{1-\gamma}{1-\gamma^{K}} P_{T}\right) \gamma^{k-1}, \quad k=1,2, \ldots, K
$$

where

$$
\gamma=1 /\left(1+P_{T} \frac{\beta}{\eta}\right)^{1 / K} .
$$

Using (29) and (27), $\beta / \eta$ in (32) is calculated as

$$
\begin{aligned}
\frac{\beta}{\eta}=\frac{T_{c}^{2}}{M N L \sigma^{2} T} \sum_{m=1}^{M} & \sum_{p=1}^{N} \sum_{i=1}^{L} \sum_{\substack{l=0 \\
l \neq i-1}}^{L-1}\left|h_{p, m, l}\right|^{2} \\
& \times(G-|l-(i-1)|) .
\end{aligned}
$$

If we assume that $G \gg L$, (33) may be approximated as

$$
\frac{\beta}{\eta} \cong \frac{T_{c}^{2} G(L-1)}{M N L \sigma^{2} T} \sum_{m=1}^{M} \sum_{p=1}^{N} \sum_{l=0}^{L-1}\left|h_{p, m, l}\right|^{2} \triangleq \rho .
$$

Note that $\gamma$ in (32) is the power ratio between the two adjacent code powers, i.e., $\gamma=P_{k+1} / P_{k}$ for $k=1,2, \ldots, K-1$. From (31), it can be seen that the code power values are determined by the total given transmit power $P_{T}$ and the power ratio $\gamma$, whose statistics will be studied in Section IV-D.

2) Space Domain Power Allocation (Second Stage):

Based on the code power values calculated in the first stage, the space domain power components for each code, $\left\{P_{k, m} \mid k=1,2, \ldots, K ; m=1,2, \ldots, M\right\}$, are computed in a recursive manner. Essentially, this procedure follows the joint power allocation algorithm described in Section IV-A, except for the reference SINR adjustment in (21i) and some procedures in Loop 1 for adaptive code power calculation. That is, it uses the Steps 2-5, (21k) in Step 7, and Step 8, when the code power values in (31) is used for $P_{k_{0}}(a)$ 's $\left(k_{0}=\right.$
$1,2, \ldots, K)$ in $(21 \mathrm{k})$ and $a$ is set to one. Compared to the joint power allocation, this space domain power allocation does not need the iterative procedure for code power adaptation. As a result, two-stage power allocation performs the computation of code domain power values (at a time) only once, and space domain power values are calculated recursively in the reverse order of code index. Thus, its complexity becomes lower than that of the joint power allocation, which may take a lot of iterations for the code power adaptation until the total power constraint is satisfied. Furthermore, note that all values for the nulling vector and detection ordering, calculated in the two-stage power allocation procedure, can be used in the detection procedure described in Section III-B without repeating calculations. Thus, compared to the computational complexity of the detection procedure, the only additional computations for the two-stage power allocation are those of (31) and (21k), which can be negligible.

Note that the two-stage power allocation derived in this subsection makes the post-detection SINRs become equal for all the substreams in the same code domain, however, the postdetection SINRs between the data substreams in the different code domains may be different.

\section{Variable and Constant Power Ratio (PR) Schemes}

The joint and two-stage schemes described in Section IV-A and IV-B respectively, require the feedback information for all the transmit power components $P_{k, m}$ 's $(k=$ $1,2, \ldots, K ; m=1,2, \ldots, M)$ from the receiver to the transmitter. As the number of transmit antennas and spreading codes increases, the amount of feedback information increases. Due to the limited feedback channel bandwidth and the resulting feedback delay, the large amount of feedback information may cause the performance degradation in practical systems. As shown in (31), for given some channel states, the code domain power allocation requires only "single" power ratio value $\gamma$ for the feedback information, since the given total transmit power $P_{T}$ is known at the transmitter. Thus, it may be desirable to allocate the different transmit powers in the code domain only as in (31), with the space domain powers distributed equally, in order to reduce the amount of feedback information. In this case, the power allocation may be written as

$$
\begin{aligned}
P_{k, m}= & \frac{1}{M}\left(\frac{1-\gamma}{1-\gamma^{K}} P_{T}\right) \gamma^{k-1}, \\
& k=1,2, \ldots, K ; \quad m=1,2, \ldots, M
\end{aligned}
$$

where $\gamma=\left(1+P_{T} \rho\right)^{-1 / K}$. We refer to this scheme as the variable PR scheme, in which the power ratio $\gamma$ varies with the instantaneous channel gains as shown in (34).

The ratio factor $\rho$ in (34) is an important parameter determining the power ratio $\gamma$ for the variable PR scheme. The expectation of $\rho$ in (34) with respect to the fading channel amplitudes $\left\{\left|h_{p, m, l}\right|\right\}$, may be calculated as

$$
E[\rho]=\frac{T(L-1)}{G L \sigma^{2}} \Omega_{\mathrm{sum}} \triangleq \rho_{C}
$$

where $\Omega_{\text {sum }} \triangleq \sum_{l=0}^{L-1} \Omega_{l}=\sum_{l=0}^{L-1} E\left[\left|h_{p, m, l}\right|^{2}\right]$. From the law of large numbers [14], it can be seen that as $M N$ 
goes to infinity, $\rho$ in (34) converges to a finite value $\rho_{C}$ in (36), since all $\left|h_{p, m, l}\right|^{2}$ s for a particular path index $l$ are assumed to be independent and identically distributed (i.i.d.) random variables with finite mean $\Omega_{l}$ for all $p$ and $m$. From this relationship between $\rho$ and $\rho_{C}$, we propose the power allocation expressed as

$$
\begin{aligned}
P_{k, m}= & \frac{1}{M}\left(\frac{1-\gamma_{C}}{1-\gamma_{C}^{K}} P_{T}\right) \gamma_{C}^{k-1}, \\
& \quad k=1,2, \ldots, K ; \quad m=1,2, \ldots, M
\end{aligned}
$$

where $\gamma_{C}=\left(1+P_{T} \rho_{C}\right)^{-1 / K}$, for the case of large number of antennas. This power allocation in (37) is the same as that in (35), except that a parameter $\rho$ in (34) is replaced by $\rho_{C}$ in (36). We refer to this scheme as the constant PR scheme. Note that the constant PR scheme uses the power ratio determined not by the instantaneous channel conditions but by the longterm statistical properties of the fading channel amplitudes. Thus, the feedback information of this power ratio is not necessary to adapt to the varying channel conditions, if longterm channel statistics is not changed. As a result, the constant PR scheme provides the significantly "reduced feedback rate" over the time-varying fading channels.

It should be noted that this small feedback overhead becomes possible since all received multicode signals go though the same channel response. In [11] and the references therein, it has been shown that a geometric distribution of "received" powers is required to have equal SINR for SIC in CDMA systems. However, the effect of fading is not considered in [11]. When different fading channel responses for different user streams are considered, the geometric power distribution in [11] can not be applied to the transmit power, and may not lead to the reduced amount of feedback information.

\section{Statistics of Code Power Ratio}

As mentioned above, the difference between the code power ratios of the variable and constant PR schemes tends to decrease, as the number of transmit and/or receive antennas increases. Thus, it can be expected that the performance of the constant PR scheme may degrade, compared to that of the variable PR scheme, when the number of antennas is small. In this subsection, the statistical properties of the code power ratio $\gamma$ in (35) is studied, in order to obtain insights into the effects of $\gamma$ on the performance difference between the variable and constant PR schemes. First, the probability density function (pdf) of $\gamma$ is derived. The power ratio $\gamma$ in (35) can be rewritten as

$$
\gamma=\frac{1}{(1+A x)^{1 / K}}
$$

where $A=P_{T} T(L-1) /\left(G L \sigma^{2}\right)$ is a constant and $x=$ $(M N)^{-1} \sum_{m=1}^{M} \sum_{p=1}^{N} \sum_{l=0}^{L-1}\left|h_{p, m, l}\right|^{2}$ is a random variable. If we assume that exponential decay rate $\delta$ in (5) is not zero, $x$ is a sum of $L$ independent and nonidentically distributed central Chi-square random variables, each with $2 M N$ degrees of freedom. The pdf of $x$ can be derived using the partialfraction expansion of the characteristic function, which may be expressed as [14], [15]

$$
\Phi_{x}(s)=\prod_{l=0}^{L-1} \frac{1}{\left(1-s \Omega_{l}^{\prime}\right)^{M N}}=\sum_{l=0}^{L-1} \sum_{n=1}^{M N} \frac{B_{l, n}}{\left(1-s \Omega_{l}^{\prime}\right)^{n}}
$$

where $s=\sqrt{-1} \cdot w, \Omega_{l}^{\prime}=\Omega_{l} / M N(l=0,1, \ldots, L-1)$, $\Omega_{l}^{\prime} \neq \Omega_{i}^{\prime}$ for $l \neq i$, and the coefficients $B_{l, n}$ 's are computed from the equation

$$
\begin{gathered}
B_{l, M N-j}=\left.\frac{1}{j !\left(-\Omega_{l}^{\prime}\right)^{j}} \frac{d^{j}}{d s^{j}}\left[\left(1-s \Omega_{l}^{\prime}\right)^{M N} \Phi_{x}(s)\right]\right|_{s=1 / \Omega_{l}^{\prime}}, \\
j=0,1, \ldots, M N-1 .
\end{gathered}
$$

It can be easily seen that $B_{0, M N}=1$ and $B_{0, n}=0$ for $n \leq M N-1$, when $L=1$. After some tedious manipulations, it can be shown that the closed-form solution of (40) for $L \geq 2$ is calculated as

$$
\begin{aligned}
& B_{l, M N-j}=\frac{1}{\left(-\Omega_{l}^{\prime}\right)^{j} \prod_{i=0, i \neq l}^{L-1}\left(1-\Omega_{i}^{\prime} / \Omega_{l}^{\prime}\right)^{M N}} \times \\
& \sum_{\substack{u_{0}+u_{1}+\cdots+u_{L-1}=j \\
u_{i} \neq u_{l}}} \prod_{\substack{i=0 \\
i \neq l}}^{L-1}\left(\begin{array}{c}
M N+u_{i}-1 \\
u_{i}
\end{array}\right)\left(\frac{\Omega_{i}^{\prime}}{1-\Omega_{i}^{\prime} / \Omega_{l}^{\prime}}\right)^{u_{i}} \text {, } \\
& L \geq 2
\end{aligned}
$$

where $u_{i}$ 's are nonnegative integers. This result in (41) is proved by the mathematical induction in Appendix B. Then, the inverse transform of the characteristic function in (39) yields the pdf of $x$ as

$$
f(x)=\sum_{l=0}^{L-1} \sum_{n=1}^{M N} B_{l, n} \frac{x^{n-1}}{\left(\Omega_{l}^{\prime}\right)^{n}(n-1) !} \exp \left(-\frac{x}{\Omega_{l}^{\prime}}\right), \quad x \geq 0 .
$$

Consequently, the pdf of $\gamma, f(\gamma)$ may be calculated as

$$
\begin{aligned}
f(\gamma)= & f(x) /|d \gamma / d x|=(K / A)(1+A x)^{1+1 / K} f(x) \\
= & (K / A) \gamma^{-K-1} \sum_{l=0}^{L-1} \sum_{n=1}^{M N} B_{l, n} \frac{\left(\left(\gamma^{-K}-1\right) / A\right)^{n-1}}{\left(\Omega_{l}^{\prime}\right)^{n}(n-1) !} \\
& \times \exp \left(-\frac{\gamma^{-K}-1}{A \Omega_{l}^{\prime}}\right), \quad \gamma \geq 0 .
\end{aligned}
$$

Given the pdf $f(\gamma)$, the mean and the variance of $\gamma$, respectively, may be calculated as $E[\gamma]=\int_{0}^{\infty} \gamma f(\gamma) d \gamma$ and $\operatorname{var}[\gamma]=\int_{0}^{\infty} \gamma^{2} f(\gamma) d \gamma-\left[\int_{0}^{\infty} \gamma f(\gamma) d \gamma\right]^{2}$, whose closedform solutions may be difficult to find. As a simple approach for approximate closed-form solutions, we use the method of expansion in differences in [16], and the results may be found $\mathrm{as}^{3}$

$$
\begin{gathered}
E[\gamma] \cong-V\left(\mu_{x}\right)+V\left(\mu_{x}+\frac{1}{\sqrt{2}} \sigma_{x}\right)+V\left(\mu_{x}-\frac{1}{\sqrt{2}} \sigma_{x}\right) \\
\operatorname{var}[\gamma] \cong \frac{1}{2}\left(V\left(\mu_{x}+\frac{1}{\sqrt{2}} \sigma_{x}\right)-V\left(\mu_{x}-\frac{1}{\sqrt{2}} \sigma_{x}\right)\right)^{2}
\end{gathered}
$$

where $V(x)=(1+A x)^{-1 / K}, \mu_{x}=\sum_{l=0}^{L-1} \Omega_{l}$ is the mean, and $\sigma_{x}^{2}=(1 / M N) \sum_{l=0}^{L-1} \Omega_{l}^{2}$ is the variance of the random

\footnotetext{
${ }^{3}$ The difference factor $h$ in [16] is set to $\sigma_{x} / \sqrt{2}$ in (44) and (45).
} 


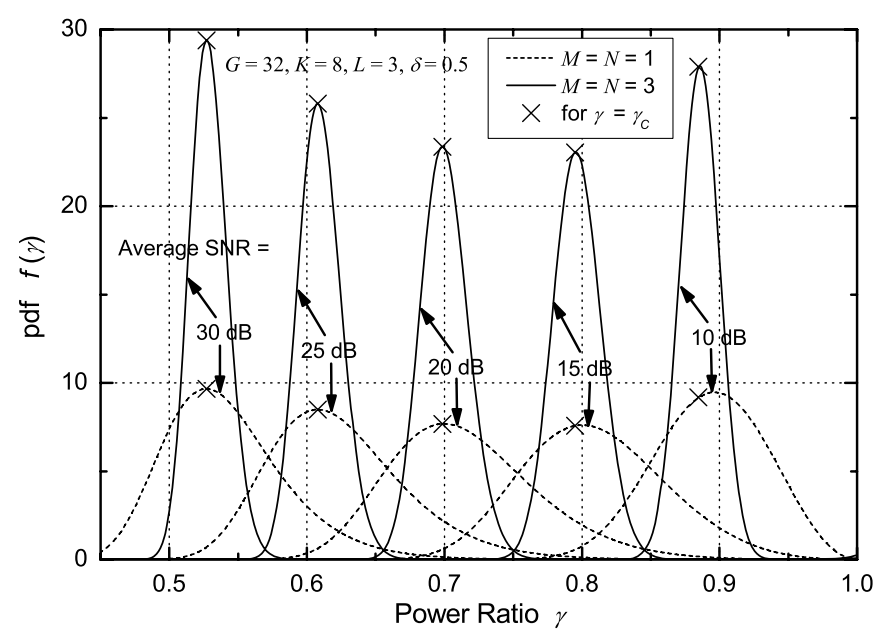

Fig. 3. Pdf of $\gamma$ for the two cases of $M=N=1$ (SISO case) and $M=N$ $=3$ (MIMO case).

variable $x$ in (38). As expected, it can be seen that the approximate mean in (44) goes to $\gamma_{C}$ in (37), and the approximate variance in (45) approaches zero, as $M N$ increases.

In Fig. 3, the pdf $f(\gamma)$ in (43) is illustrated for the two cases of $M=N=1$, i.e., single-input single-output (SISO) case, and $M=N=3$ (MIMO case) with various average SNR values, when the spreading gain $G=32$, the number of codes $K=8$, the number of multipaths $L=3$, exponential decay rate of the MIP $\delta=0.5$, and $\Omega_{\text {sum }}$ is set to 1 . Furthermore, for each pdf curve, $f(\gamma)$ at $\gamma=\gamma_{C}$ is represented by a symbol. The average SNR is defined to be $P_{T} T /\left(K \sigma^{2}\right)$. It can be seen that $\gamma$ tends to decrease as the average SNR increases. The reason for this is that, as the SNR increases, the MCI effect on the BER performance is larger than the Gaussian noise effect, and hence the more power is allocated to the data substreams corresponding to the earlier detected code index.

Fig. 3 also shows that the pdf becomes much sharper and narrower in the MIMO case than in the SISO case. In addition, the power ratio $\gamma$ of the variable PR scheme is observed to approach the power ratio $\gamma_{C}$ of the constant PR scheme with higher probability in the MIMO case than in the SISO case. When the average SNR $=20 \mathrm{~dB}$, the mean values of $\gamma$ obtained by (44) are about 0.72 in the SISO case and 0.7 in the case of $M=N=3$, and these values are nearly the same as $\gamma_{C}$, which is about 0.7 for all two cases. On the other hand, for the same SNR value, the variance of $\gamma$ obtained by (45) is about $3 \times 10^{-3}$ in the SISO case, which is much larger than the value of about $3 \times 10^{-4}$ in the case of $M=N=3$. This indicates that the performance degradation resulting from the fixed power ratio $\gamma_{C}$ regardless of the instantaneous channel states may not be negligible with the number of antennas decreasing. It may be suggested that more effective $\gamma_{C}$ can be determined by considering higher moments as well as the mean of $\gamma \cdot{ }^{4}$ However, in the cases of $M \geq 2(M=N)$ at least, the numerical results in Section $V$ show that the average BER performance of the constant PR scheme with $\gamma_{C}$ in (37), which can be obtained by simple calculation of $\rho_{C}$ in (36), is almost the same as that of the variable PR scheme.

\footnotetext{
${ }^{4}$ We will study this topic for future research.
}

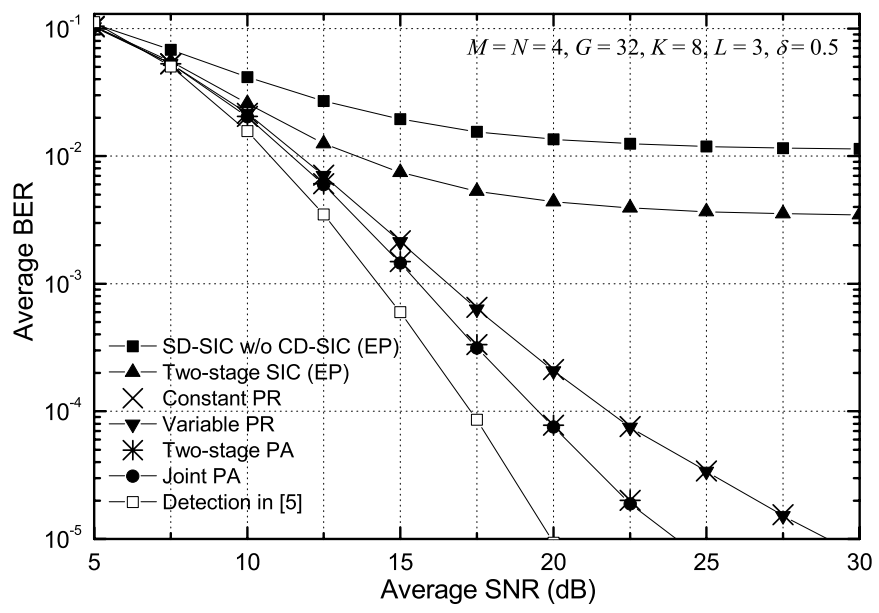

Fig. 4. BER performance comparison of power allocation schemes for $M$ $=N=4$.

\section{Numerical Results}

In this section, the performance of the transmit power allocation schemes described in Section IV are evaluated and compared with one another. The adaptive procedure in Section IV-A is used to calculate the transmit power for the joint power allocation scheme with sufficient iterations. Equations (31), (32), (34), and the adaptive procedure of space domain power allocation in Section IV-B are used for two-stage power allocation scheme. Equations (35) and (37) are used for the variable PR and constant PR schemes, respectively. The feedback channel is assumed to be an ideal error-free channel without feedback delay. Thus, the transmitter uses the same transmit power values as those calculated at the receiver. The average BER for each transmit power allocation scheme is calculated by averaging the conditional BER in (18) over sufficient number of randomly generated channel coefficients $\left\{h_{p, m, l}\right\}$, spreading sequences $\left\{c_{k, i}\right\}$, and data symbols $\left\{d_{k, m}\right\}$. Note that the decision statistics of each data symbol for each detection stage (and thus, error propagation) is considered in calculation of (18) by performing the detection process with randomly generated Gaussian noise. The spreading gain $G=32$, the number of multipaths $L=3$, the exponential decay rate of the MIP $\delta=0.5$, and $\Omega_{\text {sum }}$ is set to 1 . The average SNR is defined to be $P_{T} T /\left(K \sigma^{2}\right)$, as in Fig. 3. For spreading codes, we use the orthogonal Walsh-Hadamard codes multiplied by a common i.i.d. random complex scrambling sequence [17].

Fig. 4 compares the performance of the transmit power allocation schemes, when the number of transmit antennas $M=4$, the number of receive antennas $N=4$, and the number of spreading codes $K=8$. The scheme denoted by "SD-SIC w/o CD-SIC" in Figs. of this section allocates equal power (denoted by "EP") to all substreams and uses the SIC detection process in space domain, not in code domain, i.e., it follows the detection procedure in Section III-B except that the value of $\tilde{\mathbf{z}}_{k_{0}+1}$ in (17l) is replaced by that of $\mathbf{z}_{k_{0}+1}$ in (8). "Two-stage SIC" in Figs. is also assumed to allocate the equal power to all data substreams, while it uses the iterative two-stage SIC scheme in Section III-B. It can be seen that 
the performance of these two equal power allocation schemes suffer from irreducible error floors at average SNRs more than about $20 \mathrm{~dB}$. The BER of the SD-SIC w/o CD-SIC is shown to be about 3.3 times higher than that of the two-stage SIC at high SNR range. The joint and two-stage power allocation schemes improve the BER performance significantly over these equal power allocation schemes. It is noticeable that the performance of the joint and two-stage power allocation schemes are almost indistinguishable at all SNR ranges. Therefore, the joint power allocation scheme can be replaced by the two-stage power allocation scheme without any performance degradation but with significant savings in complexity. Fig. 4 also shows that the two-stage power allocation scheme is superior to the variable PR scheme. The SNR gains of the two-stage scheme over the variable PR scheme are about $0.9 \mathrm{~dB}$ and $2.4 \mathrm{~dB}$ at BER of $10^{-3}$ and $10^{-4}$, respectively. However, the performance difference between the variable PR and two-stage power allocation schemes is significantly small compared to that between the variable PR scheme and the two-stage SIC scheme with equal power allocation. It is also noticeable that the performance of the variable and constant PR schemes are almost indistinguishable. This indicates that, in this MIMO case of Fig. 4, the constant PR scheme can be an alternative to the variable PR scheme, and hence the feedback rate for power allocation may be reduced significantly without any performance degradation.

Fig. 4 also provides the performance of space-time detector in [5]. This detection technique uses the sequential group detection [7] for $K$ groups, and $M$ data substreams of each group are detected by V-BLAST detection algorithm. It can be seen that the space-time detector in [5] improves the performance significantly over the other schemes. However, it suffers from the cost of huge computational complexity. Using the results in [18], the computational complexity of the proposed two-stage SIC (for the despread signals) can be shown to be approximately in the order of $O\left(K M^{3} N L\right)$. That is, the V-BLAST algorithm to detect data substreams for each code has the computational complexity of $O\left(M^{3} N L\right)$ as shown in [18], and this procedure is performed $K$ times. As a result, the overall complexity order is proportional not to multiple multiplications of $K$ but to $K$ only, since the matrix dimension for inverse operation is not $K M$ (as in [5]) but $M$, and the SD-SIC procedure is iteratively performed $K$ times. On the contrary, the detection scheme in [5] requires a computational complexity of $O\left(K^{4} M^{3}\right)$ for sequential group detection processing, and that of $O\left(K M^{4}\right)$ for V-BLAST detection processing. In addition, it has a computational complexity of $O\left(K^{2} M^{2} N L\right)$ for obtaining channel matched filter outputs, which is required for the initial step of group detection technique. As a result, the overall computational complexity of the detection scheme in [5] is much higher than that of the proposed two-stage SIC scheme. In particular, compared with the two-stage SIC scheme, the sequential group detection processing increases the complexity by a factor of $K^{3} /(N L)$, which becomes very large for $K$ much larger than $N$ and $L$. Here, as mentioned in Section IV-B, it should be noted that the additional computational complexity resulting from the twostage (and PR) power allocation processing over that of the two-stage SIC scheme is negligible.

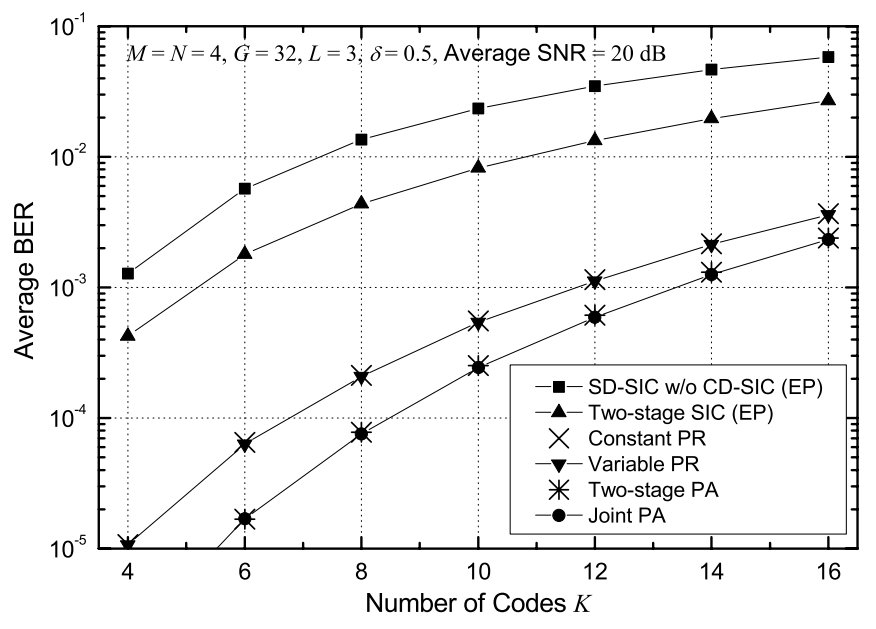

Fig. 5. BER performance versus the number of spreading codes $K$, when $M=N=4$.

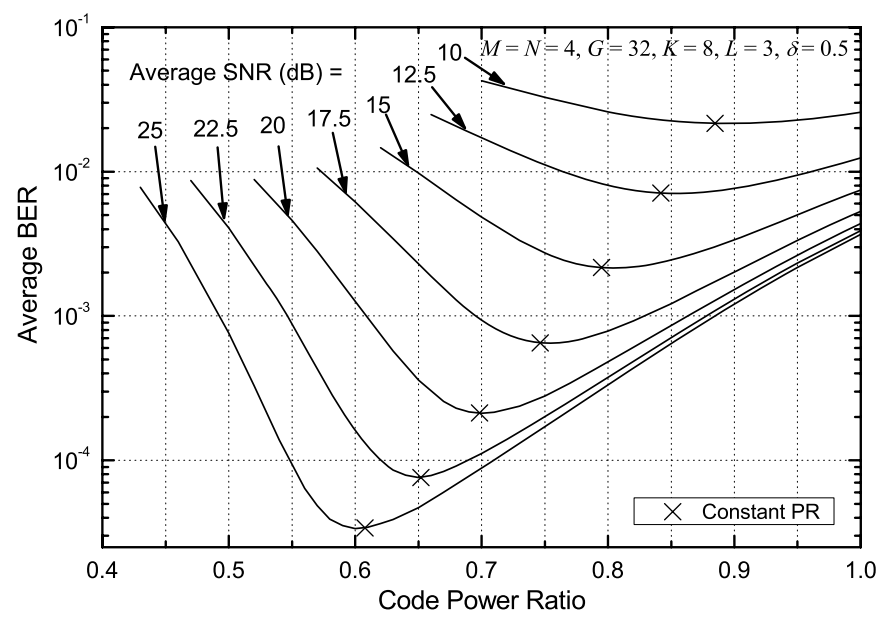

Fig. 6. BER performance versus the fixed code power ratio for various SNR values, when $M=N=4$.

Fig. 5 provides the BER performance versus the number of spreading codes $K$, when $M=N=4$ and average SNR = $20 \mathrm{~dB}$. A performance behavior similar to that in Fig. 4 is observed for all $K$. The performance difference between the equal and different power allocation schemes is seen to be larger for the smaller number of codes.

In Fig. 6, the BER performance of the power allocation scheme according to the equation (37) is illustrated with the same parameters as those in Fig. 4, when the fixed code power ratio $\gamma_{C}$ in (37) is set to arbitrary values as well as the value by the constant PR scheme. The code power ratio by the constant PR scheme is shown to provide almost the best performance for all given fixed power ratios.

Fig. 7 shows the performance comparison of the transmit power allocation schemes, when $M=N=1$ and $K=8$. As opposed to the indistinguishable performance difference between the variable and constant PR schemes in the MIMO case of Fig. 4, the constant PR scheme is seen to be significantly inferior to the variable PR scheme in this SISO case. This indicates that, as discussed in Section IV-D, the SISO case does not provide high probability of $\gamma$ approaching $\gamma_{C}$, and the 


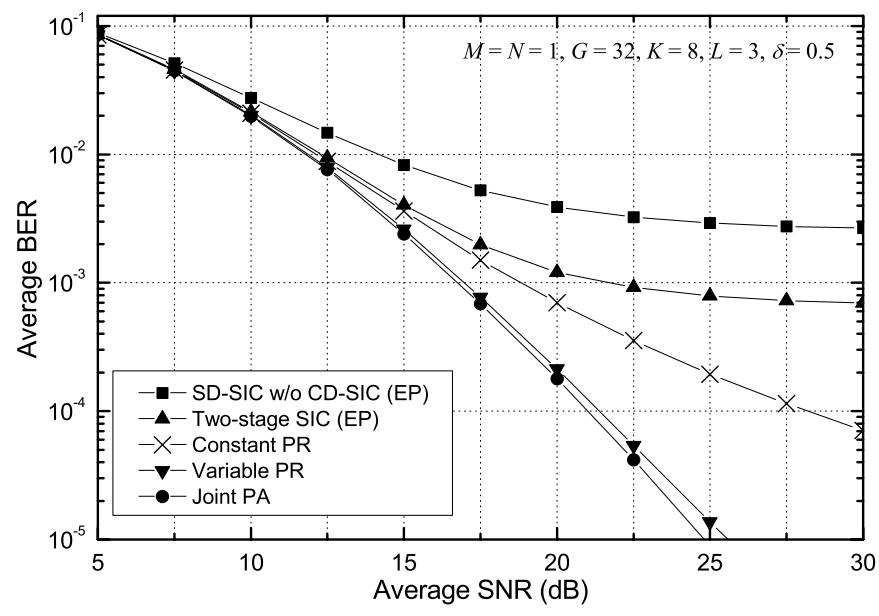

Fig. 7. BER performance comparison of power allocation schemes for $M$ $=N=1$ (SISO case).

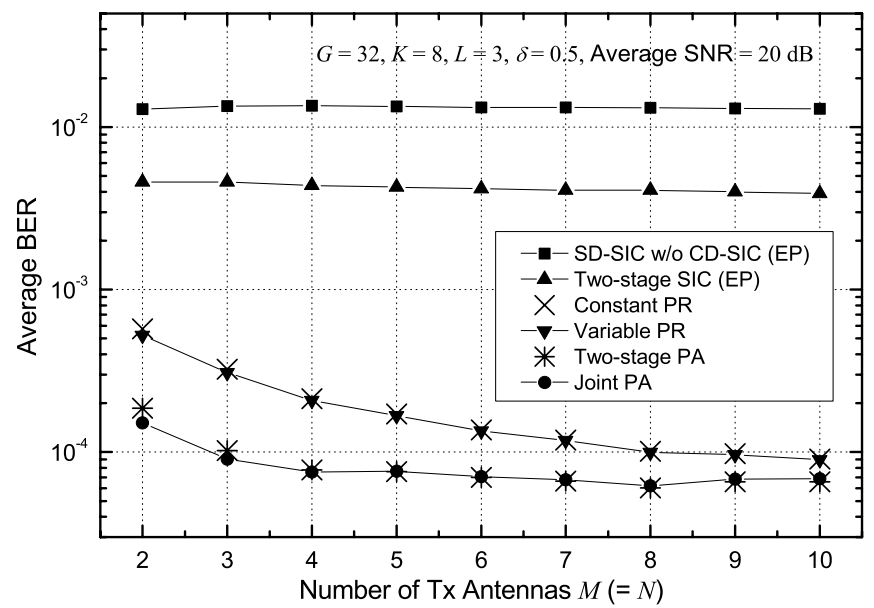

Fig. 8. Effects of the number of antennas on the BER performance of power allocation schemes, when $M=N \geq 2$.

fixed power ratio $\gamma_{C}$ regardless of the instantaneous channel states may result in the significant performance degradation.

The effects of the number of antennas in the MIMO cases of $M=N(\geq 2)$ on the BER performance are shown in Fig. 8, when $K=8$ and the average $\mathrm{SNR}=20 \mathrm{~dB}$. It can be seen that the performance of constant PR scheme is almost the same as that of variable PR scheme. Furthermore, as the number of antennas increases, the performance of variable and constant PR schemes is found to approach that of the two-stage scheme. This implies that the effects of space domain power allocation on the BER performance may be reduced with the number of transmit and receive antennas increasing, since increased diversity effects resulting from more antennas and multipaths make the performance difference between space domain substreams become smaller. Hence, for the case of a sufficiently large number of transmit and receive antennas, the simple constant PR scheme can be an alternative to the twostage power allocation scheme with negligible performance degradation.

As mentioned before, the results in this section assume that the channel estimation at the receiver is perfect and the feedback channel is an ideal error-free channel without feedback delay. Future work is needed to investigate the sensitivities of the performance to channel estimation errors and nonideal feedback channel.

\section{Conclusions}

In this paper, we have presented a simple two-stage SIC detection scheme for a multicode MIMO system, and developed the transmit power allocation schemes to improve error rate performance for this detection process. The joint power allocation has been developed to make the post-detection SINR become the same for all substreams in both space and code domains. A computationally efficient two-stage power allocation scheme has also been derived, and found to provide almost the same BER performance as the joint power allocation scheme. The variable and constant PR schemes have been developed to reduce the feedback overhead. In particular, the constant PR scheme has been shown to achieve significantly reduced feedback rate. It has also been found that the performance of the variable and constant PR schemes are almost indistinguishable in MIMO systems, and those approach the performance of the two-stage power allocation scheme, as the number of transmit and receive antennas increases.

\section{APPENDIX A \\ DERIVATION OF (31)}

In this Appendix, we derive the code power allocation in (31). From (30), we obtain

$\alpha P_{k_{0}}=\Gamma^{\mathrm{CD}}\left(\beta \sum_{k=k_{0}+1}^{K} P_{k}+\eta\right), \quad k_{0}=1,2, \ldots, K$.

Using (A.1), it can be shown that

$$
\alpha P_{k_{0}}-\alpha P_{k_{0}-1}=-\Gamma^{\mathrm{CD}} \beta P_{k_{0}}, \quad k_{0} \geq 2 .
$$

Thus, we obtain

$$
P_{k_{0}} / P_{k_{0}-1}=\alpha /\left(\alpha+\beta \Gamma^{\mathrm{CD}}\right) \triangleq \gamma, \quad k_{0} \geq 2
$$

i.e.,

$$
P_{k_{0}}=P_{1} \gamma^{k_{0}-1}, \quad k_{0}=1,2, \ldots, K .
$$

From (A.4) and the total power constraint in (3), it follows that

$$
\sum_{k=1}^{K} P_{k}=\frac{P_{1}\left(1-\gamma^{K}\right)}{1-\gamma}=P_{T}
$$

Substituting one for $k_{0}$ in (A.1) and using $\sum_{k=2}^{K} P_{k}=P_{T}-P_{1}$ gives $P_{1}=\Gamma^{\mathrm{CD}}\left(\beta P_{T}+\eta\right) /\left(\alpha+\beta \Gamma^{\mathrm{CD}}\right)$. By substituting this result and $\gamma$ in (A.3), respectively, into $P_{1}$ and $\gamma$ (not $\gamma^{K}$ ) in (A.5), we can find the power ratio $\gamma$ as

$$
\gamma=1 /\left(1+P_{T} \frac{\beta}{\eta}\right)^{1 / K}
$$

The equations (A.5) and (A.6) lead to the code power allocation in (31). 


\section{APPENDIX B \\ PROOF OF (41)}

In this Appendix, we prove that the closed-form solution of (40) is found as (41). We first define $B_{l, M N-j}$ in (40) for $L=m(m \geq 2)$ as $B_{l, M N-j}(m)(l=0,1, \ldots, m-1$; $j=0,1, \ldots, M N-1)$. For $L=2, B_{l, M N-j}(m)$ is calculated as

$$
\begin{gathered}
B_{l, M N-j}(2)=\left.\frac{1}{j !\left(-\Omega_{l}^{\prime}\right)^{j}} \frac{d^{j}}{d s^{j}} \frac{1}{\left(1-s \Omega_{i}^{\prime}\right)^{M N}}\right|_{s=1 / \Omega_{l}^{\prime}} \\
=\left(\begin{array}{c}
M N+j-1 \\
j
\end{array}\right)\left(-\frac{\Omega_{i}^{\prime}}{\Omega_{l}^{\prime}}\right)^{j} /\left(1-\frac{\Omega_{i}^{\prime}}{\Omega_{l}^{\prime}}\right)^{M N+j}
\end{gathered}
$$

where $l, i=0,1$ and $l \neq i$, which can also be derived from [15, Appendix B]. It can be easily shown that (B.1) corresponds to (41) in the case of $L=2$, and hence (41) holds for $L=2$. Now we assume that (41) holds for $L=m$ $(m \geq 2)$, i.e.,

$$
\begin{aligned}
& B_{l, M N-j}(m) \\
& \left.\triangleq \frac{1}{j !\left(-\Omega_{l}^{\prime}\right)^{j}} \frac{d^{j}}{d s^{j}}\left(\prod_{i=0, i \neq l}^{m-1} \frac{1}{\left(1-s \Omega_{i}^{\prime}\right)^{M N}}\right)\right|_{s=1 / \Omega_{l}^{\prime}} \\
& =\frac{1}{\left(-\Omega_{l}^{\prime}\right)^{j} \prod_{i=0, i \neq l}^{m-1}\left(1-\Omega_{i}^{\prime} / \Omega_{l}^{\prime}\right)^{M N}} \times \\
& \sum_{\substack{\left.u_{0}+u_{1}+\cdots+u_{m-1}=j \\
u_{i} \neq u_{l} \\
l=0,1, \ldots, m-1 ; j=0,1, \ldots, M N-1 . \quad \text { (B. } 2\right)}}^{\prod_{\substack{i=0 \\
i \neq l}}^{m-1}\left(\begin{array}{c}
M N+u_{i}-1 \\
u_{i}
\end{array}\right)\left(\frac{\Omega_{i}^{\prime}}{1-\Omega_{i}^{\prime} / \Omega_{l}^{\prime}}\right)^{u_{i}},}
\end{aligned}
$$

For $l \leq m-1, B_{l, M N-j}(m+1)$ is calculated as

$$
\begin{aligned}
B_{l, M N-j}(m+1) & \frac{1}{j !\left(-\Omega_{l}^{\prime}\right)^{j}} \frac{d^{j}}{d s^{j}}\left[\left(\prod_{i=0, i \neq l}^{m-1} \frac{1}{\left(1-s \Omega_{i}^{\prime}\right)^{M N}}\right)\right. \\
& \left.\times\left(\frac{1}{\left(1-s \Omega_{m}^{\prime}\right)^{M N}}\right)\right]\left.\right|_{s=1 / \Omega_{l}^{\prime}} \\
= & \frac{1}{j !\left(-\Omega_{l}^{\prime}\right)^{j}} \sum_{k=0}^{j}\left(\begin{array}{l}
j \\
k
\end{array}\right)\left(\frac{d^{k}}{d s^{k}} \prod_{i=0, i \neq l}^{m-1} \frac{1}{\left(1-s \Omega_{i}^{\prime}\right)^{M N}}\right) \\
& \times\left.\left(\frac{d^{j-k}}{d s^{j-k}} \frac{1}{\left(1-s \Omega_{m}^{\prime}\right)^{M N}}\right)\right|_{s=1 / \Omega_{l}^{\prime}} .
\end{aligned}
$$

Using the definition of $B_{l, M N-j}(m)$ in (B.2) and finding the $(j-k)$ th derivative of $\left(1-s \Omega_{m}^{\prime}\right)^{-M N}$ with respect to $s,($ B.3) becomes

$$
\begin{aligned}
B_{l, M N-j}(m+1) & \frac{1}{j !\left(-\Omega_{l}^{\prime}\right)^{j}} \sum_{k=0}^{j}\left(\begin{array}{l}
j \\
k
\end{array}\right) k !\left(-\Omega_{l}^{\prime}\right)^{k} B_{l, M N-k}(m) \\
& \times \frac{(M N+j-k-1) !}{(M N-1) !} \frac{\left(\Omega_{m}^{\prime}\right)^{j-k}}{\left(1-\Omega_{m}^{\prime} / \Omega_{l}^{\prime}\right)^{M N+j-k}} \\
= & \frac{1}{\left(-\Omega_{l}^{\prime}\right)^{j}\left(1-\Omega_{m}^{\prime} / \Omega_{l}^{\prime}\right)^{M N}} \sum_{k=0}^{j}\left(-\Omega_{l}^{\prime}\right)^{k} B_{l, M N-k}(m)
\end{aligned}
$$

$$
\times\left(\begin{array}{c}
M N+j-k-1 \\
j-k
\end{array}\right)\left(\frac{\Omega_{m}^{\prime}}{1-\Omega_{m}^{\prime} / \Omega_{l}^{\prime}}\right)^{j-k} .
$$

By substituting (B.2) with $j=k$ into $B_{l, M N-k}(m)$ in (B.4), it can be shown that

$$
\begin{gathered}
B_{l, M N-j}(m+1)=\frac{1}{\left(-\Omega_{l}^{\prime}\right)^{j} \prod_{i=0, i \neq l}^{m}\left(1-\Omega_{i}^{\prime} / \Omega_{l}^{\prime}\right)^{M N}} \times \\
\sum_{\substack{u_{0}+u_{1}+\cdots+u_{m}=j \\
u_{i} \neq u_{l}}} \prod_{\substack{i=0 \\
i \neq l}}^{m}\left(\begin{array}{c}
M N+u_{i}-1 \\
u_{i}
\end{array}\right)\left(\frac{\Omega_{i}^{\prime}}{1-\Omega_{i}^{\prime} / \Omega_{l}^{\prime}}\right)^{u_{i}} .
\end{gathered}
$$

By symmetry, (B.5) also holds for $l=m$. This result has shown that (41) holds for $L=m+1$ if it holds for $L=m$ $(m \geq 2)$. Therefore, it is concluded that (41) holds for all $L \geq 2$.

\section{REFERENCES}

[1] G. J. Foschini and M. J. Gans, "On limits of wireless communications in a fading environment when using multiple antennas," Wireless Personal Commun., vol. 6, pp. 311-335, Mar. 1998.

[2] E. Telatar, "Capacity of multi-antenna Gaussian channels," Eur. Trans. Telecommun., vol. 10, pp. 585-596, Nov. 1999.

[3] "Spreading and modulation (FDD)," 3rd Generation Partnership Project (3GPP), Tech. Spec. 25.213, V4.3.0, 2002.

[4] R. Zhang, T. T. Tjhung, H. B. Zhang, and P. He, "BER performance comparison of single code and multicode DS/CDMA channelization schemes for high rate data transmission," IEEE Commun. Lett., vol. 5, pp. 67-69, Feb. 2001.

[5] H. Huang, H. Viswanathan, and G. J. Foschini, "Multiple antennas in cellular CDMA systems: transmission, detection, and spectral efficiency," IEEE Trans. Wireless Commun., vol. 1, pp. 383-392, July 2002.

[6] J. Chen, J. Wang, and M. Sawahashi, "MCI cancellation for multicode wideband CDMA systems," IEEE J. Select. Areas Commun., vol. 20, pp. 450-462, Feb. 2002.

[7] M. K. Varanasi, "Group detection for synchronous Gaussian codedivision multiple-access channels," IEEE Trans. Inform. Theory, vol. 41, pp. 1083-1096, July 1995.

[8] P. W. Wolniansky, G. J. Foschini, G. D. Golden, and R. A. Valenzuela, "V-BLAST: an architecture for realizing very high data rates over the rich-scattering wireless channel," in Proc. URSI Int. Symp. Signals, Systems, and Electronics, Pisa, Italy, Sept. 1998, pp. 295-300.

[9] C. Kchao and G. L. Stüber, "Analysis of a direct-sequence spreadspectrum cellular radio system," IEEE Trans. Commun., vol. 41, pp. 1507-1516, Oct. 1993.

[10] M. Chase and K. Pahlavan, "Performance of DS-CDMA over measured indoor radio channels using random orthogonal codes," IEEE Trans. Veh. Technol., vol. 42, pp. 617-624, Nov. 1993.

[11] R. M. Buehrer, "Equal BER performance in linear successive interference cancellation for CDMA systems," IEEE Trans. Commun., vol. 49, pp. 1250-1258, July 2001.

[12] M. Tan and Y. Bar-Ness, "Equal BER power control for uplink MCCDMA with MMSE successive interference cancellation," IEEE Commun. Lett., vol. 8, pp. 348-350, June 2004.

[13] K. C. Hwang and K. B. Lee, "Joint transmit and receive filters design for multiple-input multiple-ouput (MIMO) systems," IEEE Trans. Wireless Commun., vol. 4, pp. 1635-1649, July 2005.

[14] J. G. Proakis, Digital Communications. New York: McGraw-Hill, 1995.

[15] R. R. Rick and L. B. Milstein, "Optimal decision strategies for acquisition of spread-spectrum signals in frequency-selective fading channels," IEEE Trans. Commun., vol. 46, pp. 686-694, May 1998.

[16] J. M. Holtzman, "A simple, accurate method to calculate spreadspectrum multiple-access error probabilities," IEEE Trans. Commun., vol. 40, pp. 461-464, Mar. 1992.

[17] V. K. N. Lau, "On the analysis of peak-to-average ratio (PAR) for IS95 and CDMA2000 systems," IEEE Trans. Veh. Technol., vol. 49, pp. 2174 2188, Nov. 2000.

[18] J. Benesty, Y. Huang, and J. Chen, "A fast recursive algorithm for optimum sequential signal detection in a BLAST system," IEEE Trans. Signal Processing, vol. 51, pp. 1722-1730, July 2003. 


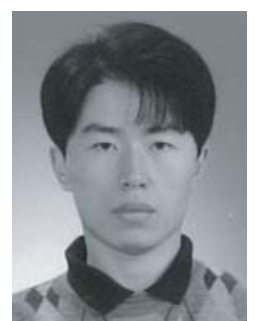

Chang Soon Park received the B.S., M.S., and $\mathrm{Ph} . D$. degrees in electrical engineering from Seoul National University, Seoul, Korea, in 2000, 2002, and 2006, respectively.

Since 2006, he has been with Samsung Advanced Institute of Technology, Korea. His current research interests include wireless communications, channel capacity, signal processing for multiple antenna systems, and multiuser MIMO wireless communications.

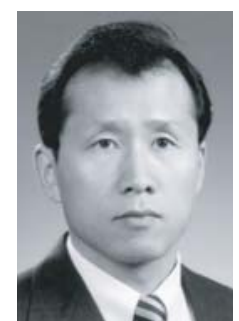

Kwang Bok Lee received the B.A.Sc. and M.Eng. degrees from the University of Toronto, Toronto, Ont., Canada, in 1982 and 1986, respectively, and the Ph.D. degree from McMaster University, Canada in 1990 .

He was with Motorola Canada from 1982 to 1985, and Motorola USA from 1990 to 1996 as a Senior Staff Engineer. At Motorola, he was involved in the research and development of wireless communication systems. He was with Bell-Northern Research, Canada, from 1989 to 1990. In March 1996, he joined the School of Electrical Engineering, Seoul National University, Seoul, Korea. Currently he is a Professor in the School of Electrical Engineering and director of Institute of New Media and Communications. He was a Vice Chair of the School of Electrical Engineering from 2000 to 2002. He has been serving as a Consultant to a number of wireless industries. His research interests include mobile communications, communication technique covering physical layer and upper layer. He holds thirteen U.S. patents and twelve Korean patents, and has a number of patents pending.

Dr. Lee was an Editor of the IEEE Journal ON SELECTED AREAS IN Communications, Wireless Series in 2001, and has been an Editor of the IEEE TRANSACTIONS ON WIRELESS COMMUNICATIONS since 2002. $\mathrm{He}$ was a Division Editor of the JOURNAL OF COMMUNICATIONS AND Networks (JCN) in 2006. And he has been the Director of JCN since 2007. And he was a co-chair of the International Conference on Communications (ICC2005) Wireless Communication Symposium, and has been a chair of IEEE Communications Society Seoul Section since 2005. He received the Best Paper Award from CDMA International Conference 2000 (CIC 2000), and the Best Teacher Award in 2003 and 2006, respectively, from College of engineering, Seoul National University. He received the Special Award from Samsung Advanced Institute of Technology in 2005. 